\title{
On the eigenfunctions of the Dirac operator on spheres and real hyperbolic spaces
}

\author{
Roberto Camporesi \\ Dipartimento di Matematica, Politecnico di Torino \\ Corso Duca degli Abruzzi 24, 10129 Torino Italy \\ and \\ Atsushi Higuchi \\ Institut für theoretische Physik, Universität Bern \\ Sidlerstrasse 5, CH-3012 Bern, Switzerland
}

February 5, 2008

\begin{abstract}
The eigenfunctions of the Dirac operator on spheres and real hyperbolic spaces of arbitrary dimension are computed by separating variables in geodesic polar coordinates. These eigenfunctions are used to derive the heat kernel of the iterated Dirac operator on these spaces. They are then studied as cross sections of homogeneous vector bundles, and a group-theoretic derivation of the spinor spherical functions and heat kernel is given based on Harish-Chandra's formula for the radial part of the Casimir operator.
\end{abstract}




\section{Introduction}

The $N$-dimensional sphere $\left(S^{N}\right)$ and the real hyperbolic space $\left(H^{N}\right)$, which are "dual" to each other as symmetric spaces [10], are maximally symmetric. This high degree of symmetry allows one to compute explicitly the eigenfunctions of the Laplacian for various fields on these spaces. These eigenfunctions can be used in studying field theory in de Sitter and anti-de Sitter spacetimes since $S^{4}$ and $H^{4}$ are Euclidean sections of these spacetimes. Also $S^{3}$ and $H^{3}$ appear as the spatial sections of cosmological models, and various field equations and their solutions on these spaces have physical applications in this context. In addition to these applications, fields on $S^{N}$ and $H^{N}$ provide concrete examples for the theory of homogeneous vector bundles, and consequences of various theorems can explicitly be worked out.

Recently the authors presented the eigenfunctions of the Laplacian for the transversetraceless totally-symmetric tensor fields [4] and for the totally-antisymmetric tensor fields ( $p$-forms) [5]. These fields were also analyzed in the light of group theory using the fact that they are cross sections of homogeneous vector bundles. As a continuation of these works we study in this paper the spinor fields satisfying the Dirac equation $\nabla \psi=i \lambda \psi$ and the heat kernel for $\nabla^{2}$ on $S^{N}$ and $H^{N}$. The paper is organized as follows. We begin by setting up the gamma matrices used in the paper in sect. 2 . Then in sect. 3 the appropriately normalized eigenfunctions of the Dirac operator on $S^{N}$ with arbitrary $N$ are presented using geodesic polar coordinates. The solutions on $S^{N}$ are expressed in terms of those on $S^{N-1}$. Then we derive the degeneracies of the Dirac operator using the spinor eigenfunctions. Next, spinor eigenfunctions on $H^{N}$ are obtained by analytically continuing those on $S^{N}$. Then they are used to derive the spinor spectral function (Plancherel measure) on $H^{N}$. In sect. 4 the results of sect. 3 are used to write down the heat kernel for the iterated Dirac operator $\nabla^{2}$ on these spaces. Sect. 5 is devoted to a group-theoretic analysis of spinor fields on $S^{N}$ and $H^{N}$. We use the fact that spinor fields on these symmetric spaces are cross sections of the homogeneous vector bundles associated to the fundamental spinor representation(s) of $\operatorname{Spin}(N)$. We first review some relevant facts about harmonic analysis for homogeneous vector bundles over compact symmetric spaces. By applying these to $S^{N}=\operatorname{Spin}(N+1) / \operatorname{Spin}(N)$, in combination with the formula for the radial part of the Casimir operator given by Harish-Chandra, we derive the spinor spherical functions and the heat kernel of the iterated Dirac operator on $S^{N}$. Then we briefly review harmonic analysis for homogeneous vector bundles over noncompact symmetric spaces, apply it to $H^{N}=\operatorname{Spin}(N, 1) / \operatorname{Spin}(N)$, and rederive some results of sect. 4 for this space. 


\section{$2 \quad \Gamma$-matrices in $N$ dimensions}

A Clifford algebra in $N$ dimensions is generated by $N$ matrices $\Gamma^{a}$ satisfying the anticommutation relations

$$
\Gamma^{a} \Gamma^{b}+\Gamma^{b} \Gamma^{a}=2 \delta^{a b} \mathbf{1}, \quad a, b=1, \ldots, N,
$$

where $\mathbf{1}$ is the unit matrix and $\delta^{a b}$ is the Kronecker symbol. It is well known that (2.1) can be satisfied by matrices of dimension $2^{\left[\frac{N}{2}\right]}$, where $\left[\frac{N}{2}\right]=N / 2$ for $N$ even, $\left[\frac{N}{2}\right]=(N-1) / 2$ for $N$ odd. We give below an inductive construction of $\Gamma^{a}$ which relates easily to the spinor representations of the orthogonal groups.

For $N=1$ we set $\Gamma^{1}=1 \in \mathbf{R}$. For $N=2$ put

$$
\Gamma^{2}=\left(\begin{array}{ll}
0 & 1 \\
1 & 0
\end{array}\right), \quad \Gamma^{1}=\left(\begin{array}{cc}
0 & i \\
-i & 0
\end{array}\right)
$$

where $i=\sqrt{-1}$. For $N=3$ we add to $\Gamma^{1}$ and $\Gamma^{2}$ above the matrix

$$
\Gamma^{3}=(-i) \Gamma^{1} \Gamma^{2}=\left(\begin{array}{cc}
1 & 0 \\
0 & -1
\end{array}\right)
$$

For $N=4$ let

$$
\Gamma^{4}=\left(\begin{array}{cc}
0 & \mathbf{1} \\
\mathbf{1} & 0
\end{array}\right), \quad \Gamma^{j}=\left(\begin{array}{cc}
0 & i \tilde{\Gamma}^{j} \\
-i \tilde{\Gamma}^{j} & 0
\end{array}\right), \quad j=1,2,3,
$$

where 1 is the $2 \times 2$ unit matrix, and the matrices $\tilde{\Gamma}^{j}(j=1,2,3)$ satisfy the Clifford algebra for $N=3$ and are given by the right-hand sides of (2.2)-(2.3). For $N=5$ we add to $\Gamma^{j}(j=1, \ldots, 4)$ given in $(2.4)$ the matrix

$$
\Gamma^{5}=(-i)^{2} \Gamma^{1} \Gamma^{2} \Gamma^{3} \Gamma^{4}=\left(\begin{array}{cc}
\mathbf{1} & 0 \\
0 & \mathbf{- 1}
\end{array}\right)
$$

The general pattern is now clear.

Case 1. $N$ even. Let $\Gamma^{a}=\left(\Gamma^{j}, \Gamma^{N}\right)$ denote the following set of matrices of dimension $2^{N / 2}$ :

$$
\Gamma^{N}=\left(\begin{array}{ll}
0 & \mathbf{1} \\
\mathbf{1} & 0
\end{array}\right), \quad \Gamma^{j}=\left(\begin{array}{cc}
0 & i \tilde{\Gamma}^{j} \\
-i \tilde{\Gamma}^{j} & 0
\end{array}\right), \quad j=1, \ldots, N-1,
$$

where 1 is the unit matrix of dimension $2^{N / 2-1}$, and the matrices $\tilde{\Gamma}^{j}$ (also of dimension $2^{N / 2-1}$ ) satisfy the Clifford algebra relations in $N-1$ dimensions,

$$
\tilde{\Gamma}^{j} \tilde{\Gamma}^{k}+\tilde{\Gamma}^{k} \tilde{\Gamma}^{j}=2 \delta^{j k}, \quad j, k=1, \ldots, N-1 .
$$


Then (2.1) is immediately verified. This representation generalizes to $N$ even the ordinary spinor representation of gamma matrices in four dimensions, where all $\gamma_{a}$ 's are "offdiagonal" and $\gamma_{5}$ is diagonal.

The $\frac{1}{2} N(N-1)$ matrices

$$
\Sigma^{a b} \equiv \frac{1}{4}\left[\Gamma^{a}, \Gamma^{b}\right], \quad a, b=1, \ldots, N
$$

satisfy the $S O(N)$ commutation rules

$$
\left[\Sigma^{a b}, \Sigma^{c d}\right]=\delta^{b c} \Sigma^{a d}-\delta^{a c} \Sigma^{b d}-\delta^{b d} \Sigma^{a c}+\delta^{a d} \Sigma^{b c}
$$

and generate a $2^{N / 2}$-dimensional representation of $\operatorname{Spin}(N)$, the double cover of $S O(N)$ (for $N>2 \operatorname{Spin}(N)$ is also the universal covering group). The commutator of $\Sigma^{a b}$ and $\Gamma^{c}$ is then

$$
\left[\Sigma^{a b}, \Gamma^{c}\right]=\delta^{b c} \Gamma^{a}-\delta^{a c} \Gamma^{b}
$$

It is easily seen that the matrix $\boldsymbol{\eta}=\Gamma^{1} \Gamma^{2} \cdots \Gamma^{N}$ anticommutes with each of the $\Gamma^{a}$ 's, and commutes with each of the $\operatorname{Spin}(N)$ generators $\Sigma^{a b}$. It can be shown by induction that

$$
\boldsymbol{\eta}=i^{N / 2}\left(\begin{array}{cc}
\mathbf{1} & 0 \\
0 & -\mathbf{1}
\end{array}\right) .
$$

Since $\boldsymbol{\eta}$ is nontrivial it follows that the representation $\tau$ of $\operatorname{Spin}(N)$ with generators $\Sigma^{a b}$ must be reducible. From (2.6) we find

$$
\Sigma^{a b}=\left(\begin{array}{cc}
\Sigma_{+}^{a b} & 0 \\
0 & \Sigma_{-}^{a b}
\end{array}\right),
$$

where $\Sigma_{+}^{a b}$ and $\Sigma_{-}^{a b}$ both satisfy $(2.9)$ and are given by

$$
\begin{aligned}
\Sigma_{ \pm}^{j k} & =\tilde{\Sigma}^{j k} \equiv \frac{1}{4}\left[\tilde{\Gamma}^{j}, \tilde{\Gamma}^{k}\right], \\
\Sigma_{ \pm}^{j N} & =-\Sigma_{ \pm}^{N j}= \pm \frac{i}{2} \tilde{\Gamma}^{j}, \quad j, k=1, \ldots, N-1,
\end{aligned}
$$

It can be shown that the matrices $\Sigma_{+}^{a b}$ and $\Sigma_{-}^{a b}$ generate the irreducible representations of $\operatorname{Spin}(N)$ with highest weights $\tau_{+}$and $\tau_{-}$respectively, where

$$
\tau_{ \pm}=\left(\frac{1}{2}, \frac{1}{2}, \ldots, \frac{1}{2}, \pm \frac{1}{2}\right)
$$

in the standard Cartan-Weyl labeling scheme. Since these weights are fundamental (see [1] p.224), $\tau_{+}$and $\tau_{-}$are called the two fundamental spinor representations of $\operatorname{Spin}(N)$ (of dimension $2^{N / 2-1}$ each). Thus $\tau=\tau_{+} \oplus \tau_{-}$and a spinor in $N$ dimensions ( $N$ even) is reducible with respect to the orthogonal group. 
In a similar way, the matrices $\tilde{\Sigma}^{j k}$ given by (2.13) generate the unique fundamental spinor representation $\sigma$ of $\operatorname{Spin}(N-1)$ (see Case 2 below), also of dimension $2^{N / 2-1}$, and one has the branching rule

$$
\left.\tau_{+}\right|_{\operatorname{Spin}(N-1)}=\sigma=\left.\tau_{-}\right|_{\operatorname{Spin}(N-1)} .
$$

Case 2. $N$ odd. In this case the dimension of the $\Gamma$-matrices is $2^{(N-1) / 2}$, the same as in $N-1$ dimensions. Let $\tilde{\Gamma}^{j}(j=1, \ldots, N-1)$ be a set of matrices satisfying the Clifford algebra anticommutation relations (2.7) in $N-1$ dimensions. As observed above, the matrix

$$
\boldsymbol{\eta} \equiv \tilde{\Gamma}^{1} \tilde{\Gamma}^{2} \cdots \tilde{\Gamma}^{N-1}
$$

anticommutes with each of the $\tilde{\Gamma}^{j}$ s. Thus if we define

$$
\Gamma^{N}=\epsilon \boldsymbol{\eta}
$$

where the constant $\epsilon$ is such that $\left(\Gamma^{N}\right)^{2}=\mathbf{1}$, then the matrices

$$
\Gamma^{a}=\left(\tilde{\Gamma}^{j}, \Gamma^{N}\right)
$$

satisfy the Clifford algebra (2.1) in $N$ dimensions. It can be shown by induction that the choice $\epsilon=(-i)^{(N-1) / 2}$ gives

$$
\Gamma^{N}=\left(\begin{array}{cc}
\mathbf{1} & \mathbf{0} \\
\mathbf{0} & -\mathbf{1}
\end{array}\right)
$$

and, as a result,

$$
\Gamma^{1} \Gamma^{2} \cdots \Gamma^{N}=i^{(N-1) / 2}
$$

Again the matrices $\Sigma^{a b}$ defined by (2.8) satisfy (2.9). This time they generate an irreducible representation of $\operatorname{Spin}(N)$, namely the unique fundamental spinor representation with highest weight

$$
\tau=\left(\frac{1}{2}, \frac{1}{2}, \ldots, \frac{1}{2}\right)
$$

(see [1] p.224). If $\sigma_{+}$and $\sigma_{-}$denote the two fundamental spinor representations of $\operatorname{Spin}(N-1)$ (see Case 1 above), we have the branching rule

$$
\left.\tau\right|_{\operatorname{Spin}(N-1)}=\sigma_{+} \oplus \sigma_{-} .
$$

Clearly the generators of $\left.\tau\right|_{\operatorname{Spin}(N-1)}$ are the matrices $\tilde{\Sigma}^{j k} \equiv \frac{1}{4}\left[\tilde{\Gamma}^{j}, \tilde{\Gamma}^{k}\right]$. 


\section{Spherical modes of the Dirac operator}

\section{$3.1 S^{N}$}

The metric on $S^{N}$ may be written in geodesic polar coordinates as

$$
d s_{N}^{2}=d \theta^{2}+f^{2}(\theta) d s_{N-1}^{2}=d \theta^{2}+f^{2}(\theta) \sum_{i, j=1}^{N-1} \tilde{g}_{i j} d \omega^{i} \otimes d \omega^{j},
$$

where $\theta$ is the geodesic distance from the origin (north-pole), $f(\theta)=\sin \theta$, and $\left\{\omega^{i}\right\}$ are coordinates on $S^{N-1}$, with metric tensor $\tilde{g}_{i j}(\omega)=<\partial / \partial \omega^{i}, \partial / \partial \omega^{j}>$. Let $\left\{\tilde{\mathbf{e}}_{j}\right\}$ be a vielbein (i.e. an orthonormal frame) on $S^{N-1}$, with anolonomy and (Levi-Civita) connection coefficients

$$
\begin{gathered}
{\left[\tilde{\mathbf{e}}_{i}, \tilde{\mathbf{e}}_{j}\right]=\sum_{k=1}^{N-1} \tilde{C}_{i j k} \tilde{\mathbf{e}}_{k},} \\
\tilde{\omega}_{i j k}=<\tilde{\nabla}_{\tilde{\mathbf{e}}_{i}} \tilde{\mathbf{e}}_{j}, \tilde{\mathbf{e}}_{k}>=\frac{1}{2}\left(\tilde{C}_{i j k}-\tilde{C}_{i k j}-\tilde{C}_{j k i}\right) .
\end{gathered}
$$

Let $\left\{\tilde{\mathbf{e}}^{j}\right\}$ be the dual coframe to $\left\{\tilde{\mathbf{e}}_{j}\right\}$. Then $d s_{N-1}^{2}=\sum_{i=1}^{N-1} \tilde{\mathbf{e}}^{i} \otimes \tilde{\mathbf{e}}^{i}$. We shall work in the geodesic polar coordinates vielbein $\left\{\mathbf{e}_{a}\right\}_{a=1, \ldots, N}$ on $S^{N}$ defined by

$$
\mathbf{e}_{N}=\partial_{\theta} \equiv \partial / \partial \theta, \quad \mathbf{e}_{j}=\frac{1}{f(\theta)} \tilde{\mathbf{e}}_{j}, \quad j=1, \ldots, N-1
$$

The only nonvanishing components of the Levi-Civita connection $\omega_{a b c}$ in the frame $\left\{\mathbf{e}_{a}\right\}$ are found to be

$$
\omega_{i j k}=\frac{1}{f} \tilde{\omega}_{i j k}, \quad \omega_{i N k}=-\omega_{i k N}=\frac{f^{\prime}}{f} \delta_{i k}, \quad i, j, k=1, \ldots, N-1,
$$

where a prime denotes differentiation with respect to the argument. Note that $\omega_{a b c}=$ $\omega_{a[b c]} \equiv \frac{1}{2}\left(\omega_{a b c}-\omega_{a c b}\right)$, as required in a vielbein for a metric connection.

Spinors are $2^{\left[\frac{N}{2}\right]}$-dimensional and are associated with orthonormal frames. Under a local rotation $\Lambda: S^{N} \rightarrow S O(N)$, which transforms the covielbein as

$$
\mathbf{e}^{a}(x) \rightarrow \mathbf{e}^{\prime a}(x)=\sum_{b} \Lambda(x)^{a}{ }_{b} \mathbf{e}^{b}(x),
$$

a spinor transforms by definition according to

$$
\psi(x) \rightarrow \psi^{\prime}(x)=S(\Lambda(x)) \psi(x)
$$

where $S(\Lambda(x))$ is either one of the two elements of $\operatorname{Spin}(N)$ (more precisely of the spin representation $\tau$ of $\operatorname{Spin}(N)$, see section 2) which correspond to $\Lambda(x) \in S O(N)$, and is determined by

$$
S(\Lambda(x))^{-1} \Gamma^{a} S(\Lambda(x))=\sum_{b} \Lambda(x)^{a}{ }_{b} \Gamma^{b} .
$$


A spin connection on $S^{N}$ is induced by the Levi-Civita connection $\boldsymbol{\omega}$. The covariant derivative $\nabla_{a} \psi$ of a spinor $\psi$ along the vielbein $\mathbf{e}_{a}$ may be written as

$$
\nabla_{a} \psi=\mathbf{e}_{a} \psi-\frac{1}{2} \omega_{a b c} \Sigma^{b c} \psi
$$

where summation over repeated indices is understood from now on. The Dirac operator is defined by $\not \psi=\Gamma^{a} \nabla_{a} \psi$. We shall now solve for the eigenfunctions of $\not \nabla$ on $S^{N}$ by separating variables in geodesic polar coordinates.

Case 1. $N$ even. Using eqs. (2.6), (2.7), (2.12), (2.13), (2.14), and (3.5), it is straightforward to derive the following expression for the Dirac operator in the vielbein (3.4):

$$
\begin{aligned}
\not \nabla \psi & =\left(\partial_{\theta}+\frac{N-1}{2} \frac{f^{\prime}}{f}\right) \Gamma^{N} \psi+\frac{1}{f} \Gamma^{i}\left(\tilde{\mathbf{e}}_{i}-\frac{1}{2} \tilde{\omega}_{i j k} \Sigma^{j k}\right) \psi \\
& =\left(\partial_{\theta}+\frac{N-1}{2} \frac{f^{\prime}}{f}\right) \Gamma^{N} \psi+\frac{1}{f}\left(\begin{array}{cc}
0 & i \tilde{\nabla} \\
-i \tilde{\nabla} & 0
\end{array}\right) \psi,
\end{aligned}
$$

where $i=\sqrt{-1}$ and

$$
\tilde{\nabla}=\tilde{\Gamma}^{j} \tilde{\nabla}_{j}=\tilde{\Gamma}^{j}\left(\tilde{\mathbf{e}}_{j}-\frac{1}{2} \tilde{\omega}_{j k l} \tilde{\Sigma}^{k l}\right)
$$

is the Dirac operator on $S^{N-1}$. We now project the first order Dirac equation

$$
\not \psi=i \lambda \psi
$$

onto the "upper" and "lower" components of $\psi$. Define

$$
\psi \equiv\left(\begin{array}{c}
\phi_{+} \\
\phi_{-}
\end{array}\right)
$$

Then

$$
\left\{\begin{array}{l}
\left(\partial_{\theta}+\frac{N-1}{2} \frac{f^{\prime}}{f}\right) \phi_{-}+\frac{1}{f} i \tilde{\nabla} \phi_{-}=i \lambda \phi_{+} \\
\left(\partial_{\theta}+\frac{N-1}{2} \frac{f^{\prime}}{f}\right) \phi_{+}-\frac{1}{f} i \tilde{\nabla} \phi_{+}=i \lambda \phi_{-} .
\end{array}\right.
$$

Eliminating $\phi_{-}\left(\right.$or $\left.\phi_{+}\right)$gives the second order equation

$$
\left[\left(\partial_{\theta}+\frac{N-1}{2} \frac{f^{\prime}}{f}\right)^{2}+\frac{1}{f^{2}} \tilde{\nabla}^{2} \pm \frac{f^{\prime}}{f^{2}} i \tilde{\nabla}\right] \phi_{ \pm}=-\lambda^{2} \phi_{ \pm},
$$

which is equivalent to the equation obtained by squaring the Dirac operator, i.e.

$$
\nabla^{2} \psi=-\lambda^{2} \psi
$$


It is well known that on a compact spin manifold $\nabla^{2}$ is negative semidefinite (the spectrum of $\not \nabla$ is purely imaginary), so that $\lambda$ is real. Now suppose that we have solved the eigenvalue equation on $S^{N-1}$, i.e.

$$
\tilde{\nabla} \chi_{l m}^{( \pm)}= \pm i(l+\rho) \chi_{l m}^{( \pm)}
$$

Here, the index $l=0,1, \ldots$, labels the eigenvalues of the Dirac operator on the $(N-1)$ sphere, and $\rho \equiv(N-1) / 2$, I while the index $m$ runs from 1 to the degeneracy $d_{l}$. Since the dimension of $\chi$ is the same as the dimension of $\phi_{ \pm}$, i.e. $2^{N / 2-1}$, one can separate variables in the following way:

$$
\begin{aligned}
{ }^{(1)} \phi_{+n l m}(\theta, \Omega) & =\phi_{n l}(\theta) \chi_{l m}^{(-)}(\Omega), \\
{ }^{(2)} \phi_{+n l m}(\theta, \Omega) & =\psi_{n l}(\theta) \chi_{l m}^{(+)}(\Omega),
\end{aligned}
$$

and similarly for the "lower" spinor $\phi_{-}$. Here $\Omega \in S^{N-1}$, and $n=0,1, \cdots$ labels the eigenvalues $-\lambda_{n, N}^{2}$ of $\not^{2}$ on $S^{N}$ and $n \geq l$ as is well known. (We shall see also that this condition arises as the requirement for the absence of singularity in the mode functions.)

Substituting (3.18) in (3.15) we obtain the following equation for the scalar functions $\phi_{n l}$ :

$$
D \phi_{n l}=-\lambda_{n, N}^{2} \phi_{n l}
$$

where $D$ is the differential operator

$$
\begin{aligned}
D & =\left(\frac{\partial}{\partial \theta}+\rho \cot \theta\right)^{2}-\frac{(l+\rho)^{2}}{\sin ^{2} \theta}+(l+\rho) \frac{\cos \theta}{\sin ^{2} \theta} \\
& =\frac{\partial^{2}}{\partial \theta^{2}}+(N-1) \cot \theta \frac{\partial}{\partial \theta}-\frac{(l+\rho)^{2}-\rho(\rho-1)}{\sin ^{2} \theta}+(l+\rho) \frac{\cos \theta}{\sin ^{2} \theta}-\rho^{2} .
\end{aligned}
$$

A simple calculation allows us to rewrite $D$ in the following form

$$
D=\left(\cos \frac{\theta}{2}\right)^{l+1}\left(\sin \frac{\theta}{2}\right)^{l}\left(D_{\cos \theta}^{\left(\frac{N}{2}+l-1, \frac{N}{2}+l\right)}-\left(l+\frac{N}{2}\right)^{2}\right) \circ\left(\cos \frac{\theta}{2}\right)^{-l-1}\left(\sin \frac{\theta}{2}\right)^{-l},
$$

where $D_{x}^{(a, b)}$ is the differential operator for the Jacobi polynomials $P_{n}^{(a, b)}(x)$,

$$
\begin{aligned}
D_{x}^{(a, b)} & =\left(1-x^{2}\right) \partial_{x}^{2}+[b-a-(a+b+2) x] \partial_{x}, \\
D_{x}^{(a, b)} P_{n}^{(a, b)}(x) & =-n(n+a+b+1) P_{n}^{(a, b)}(x) .
\end{aligned}
$$

\footnotetext{
${ }^{1}$ The spectrum of the Dirac operator on spheres is well known (see e.g. [17]). It will be clear that our procedure gives an independent proof of (3.26) by induction over the dimension $N$ (see also the remark at the end of this subsection). Eq. (3.17) (and the analogous relation (3.51)) may then be assumed as the inductive hypothesis in this proof. A group-theoretic derivation of the spectrum of $\not^{2}$ on $S^{N}$ will be given in subsections 5.1 and 5.2 .
} 
Thus, the unique regular solution to $(3.20)$ is, up to a normalization factor,

$$
\phi_{n l}(\theta)=\left(\cos \frac{\theta}{2}\right)^{l+1}\left(\sin \frac{\theta}{2}\right)^{l} P_{n-l}^{\left(\frac{N}{2}+l-1, \frac{N}{2}+l\right)}(\cos \theta),
$$

with $n-l \geq 0$ - this condition is needed for the regularity of the eigenfunctions - and with the eigenvalues

$$
\lambda_{n, N}^{2}=\left(n+\frac{N}{2}\right)^{2} .
$$

At $\theta=0$ only the modes with $l=0$ are nonzero. The functions $\phi_{n 0}$ are called spinor spherical functions.

By proceeding in a similar way with the functions $\psi_{n l}$ in 3.19 ) we find

$$
\begin{aligned}
\psi_{n l}(\theta) & =\left(\cos \frac{\theta}{2}\right)^{l}\left(\sin \frac{\theta}{2}\right)^{l+1} P_{n-l}^{\left(\frac{N}{2}+l, \frac{N}{2}+l-1\right)}(\cos \theta) \\
& =(-1)^{n-l} \phi_{n l}(\pi-\theta)
\end{aligned}
$$

They all vanish at the north pole, but for $l=0$ they are nonzero at the south pole $(\theta=\pi)$.

One can readily verify that if $\phi_{+}$satisfies (3.15) and if $\phi_{-}$is defined by the second equation of (3.14), then the first equation is automatically satisfied. In this way we can find the eigenfunctions of the first-order Dirac operator. We use the formulae

$$
\begin{aligned}
& {\left[\frac{d}{d \theta}+\frac{N-1}{2} \cot \theta-\frac{l+\frac{N-1}{2}}{\sin \theta}\right] \phi_{n l}(\theta)=-\left(n+\frac{N}{2}\right) \psi_{n l}(\theta),} \\
& {\left[\frac{d}{d \theta}+\frac{N-1}{2} \cot \theta+\frac{l+\frac{N-1}{2}}{\sin \theta}\right] \psi_{n l}(\theta)=\left(n+\frac{N}{2}\right) \phi_{n l}(\theta),}
\end{aligned}
$$

to simplify the expressions. These can be proved by using the expression of the Jacobi polynomial in terms of the hypergeometric function,

$$
P_{n}^{(\alpha, \beta)}(x)=\frac{\Gamma(n+\alpha+1)}{\Gamma(n+1) \Gamma(\alpha+1)} F\left(n+\alpha+\beta+1,-n, \alpha+1 ; \frac{1-x}{2}\right),
$$

and the raising and lowering operators for the third entry of the hypergeometric function. Thus, we find the solutions to the first-order Dirac equation to be

$$
\begin{aligned}
& \psi_{ \pm n l m}^{(-)}(\theta, \Omega)=\frac{c_{N}(n l)}{\sqrt{2}}\left(\begin{array}{c}
\phi_{n l}(\theta) \chi_{l m}^{(-)}(\Omega) \\
\pm i \psi_{n l}(\theta) \chi_{l m}^{(-)}(\Omega)
\end{array}\right), \\
& \psi_{ \pm n l m}^{(+)}(\theta, \Omega)=\frac{c_{N}(n l)}{\sqrt{2}}\left(\begin{array}{c}
i \psi_{n l}(\theta) \chi_{l m}^{(+)}(\Omega) \\
\pm \phi_{n l}(\theta) \chi_{l m}^{(+)}(\Omega)
\end{array}\right) .
\end{aligned}
$$

These spinors satisfy

$$
\not \psi_{ \pm n l m}^{(s)}= \pm i\left(n+\frac{N}{2}\right) \psi_{ \pm n l m}^{(s)}
$$


with $s= \pm$, and are required to satisfy the normalization condition

$$
\int_{S^{N}} d \Omega_{N} \psi_{+n l m}^{(s)}(\theta, \Omega)^{\dagger} \psi_{+n^{\prime} l^{\prime} m^{\prime}}^{\left(s^{\prime}\right)}(\theta, \Omega)=\delta_{n n^{\prime}} \delta_{l l^{\prime}} \delta_{m m^{\prime}} \delta_{s s^{\prime}}
$$

with an analogous relation for $\psi_{+}^{(s)} \rightarrow \psi_{-}^{(s)}$.

Suppose that the spinors $\chi_{l m}^{( \pm)}(\Omega)$ are normalized by

$$
\int_{S^{N-1}} d \Omega_{N-1} \chi_{l m}^{(s)}(\Omega)^{\dagger} \chi_{l^{\prime} m^{\prime}}^{\left(s^{\prime}\right)}(\Omega)=\delta_{l l^{\prime}} \delta_{m m^{\prime}} \delta_{s s^{\prime}}
$$

Then the normalization factor $c_{N}(n l)$ is determined by

$$
\left|c_{N}(n l)\right|^{-2}=\frac{1}{2} \int_{0}^{\pi} d \theta \sin ^{N-1} \theta\left(\phi_{n l}(\theta)^{2}+\psi_{n l}(\theta)^{2}\right) .
$$

It is clear from (3.28) that the integrals of the first and the second terms are equal. Using Ref. [8] eq. 7.391 n.1 we find

$$
\begin{aligned}
\left|c_{N}(n l)\right|^{-2} & =\int_{0}^{\pi} d \theta \sin ^{N-1} \theta \phi_{n l}(\theta)^{2} \\
& =\frac{2^{N-2}\left|\Gamma\left(\frac{N}{2}+n\right)\right|^{2}}{(n-l) !(N+n+l-1) !} .
\end{aligned}
$$

The spinor eigenfunctions with $l=0$ near $\theta=0$ are then given by

$$
\begin{aligned}
\psi_{ \pm n 0 m}^{(-)}(\theta, \Omega) & \sim\left[\frac{(N+n-1) !}{2^{N-1} n !|\Gamma(N / 2)|^{2}}\right]^{1 / 2}\left(\begin{array}{c}
\chi_{0 m}^{(-)} \\
0
\end{array}\right), \\
\psi_{ \pm n 0 m}^{(+)}(\theta, \Omega) & \sim\left[\frac{(N+n-1) !}{2^{N-1} n !|\Gamma(N / 2)|^{2}}\right]^{1 / 2}\left(\begin{array}{c}
0 \\
\chi_{0 m}^{(+)}
\end{array}\right) .
\end{aligned}
$$

The degeneracy for the eigenvalue $+i(n+N / 2)[$ or $-i(n+N / 2)]$ is given by (take $n=n^{\prime}$, $l=l^{\prime}, m=m^{\prime}, s=s^{\prime}$ in (3.35) and sum over $l, m, s$ )

$$
\begin{aligned}
D_{N}(n) & =\int_{S^{N}} d \Omega_{N} \sum_{s l m} \psi_{+n l m}^{(s)}(\theta, \Omega)^{\dagger} \psi_{+n l m}^{(s)}(\theta, \Omega) \\
& =\Omega_{N} \times \lim _{\theta \rightarrow 0} \sum_{s m} \psi_{+n 0 m}^{(s)}(\theta, \Omega)^{\dagger} \psi_{+n 0 m}^{(s)}(\theta, \Omega) \\
& =\frac{\Omega_{N}(N+n-1) !}{2^{N-1} n !|\Gamma(N / 2)|^{2}} \sum_{m}\left[\chi_{0 m}^{(+)}(\Omega)^{\dagger} \chi_{0 m}^{(+)}(\Omega)+\chi_{0 m}^{(-)}(\Omega)^{\dagger} \chi_{0 m}^{(-)}(\Omega)\right],
\end{aligned}
$$

where we have used the fact that the sum over $s, l, m$ inside the integral is constant over $S^{N}$ (this is easy to prove), so that it may be calculated for $\theta \rightarrow 0$, where only the $l=0$ term survives. The factor $\Omega_{N}$ is the volume of $S^{N}$,

$$
\Omega_{N}=\frac{2 \pi^{(N+1) / 2}}{\Gamma((N+1) / 2)} .
$$


Now the degeneracy $d_{0}$ of $+i \rho($ or $-i \rho)$ on $S^{N-1}$ (cf. (3.17)) coincides with the dimension $2^{N / 2-1}$ of the representation of $\operatorname{Spin}(N)$ labelled by $l=0$ (see section 5$)$. Thus $m=$ $1,2, \ldots, 2^{N / 2-1}$ in (3.41), and we have the identity

$$
\begin{aligned}
2^{N / 2-1} & =\sum_{m} \int d \Omega_{N-1} \chi_{0 m}^{(s)}(\Omega)^{\dagger} \chi_{0 m}^{(s)}(\Omega) \\
& =\Omega_{N-1} \sum_{m} \chi_{0 m}^{(s)}(\Omega)^{\dagger} \chi_{0 m}^{(s)}(\Omega) .
\end{aligned}
$$

From this equation and eqs. (3.41) and (3.42) we obtain

$$
D_{N}(n)=\frac{2^{N / 2}(N+n-1) !}{n !(N-1) !},
$$

where we have used the doubling formula for the Gamma function

$$
\Gamma(x) \Gamma\left(x+\frac{1}{2}\right)=\frac{\sqrt{\pi}}{2^{2 x-1}} \Gamma(2 x) .
$$

The degeneracy $D_{N}(n)$ is equal to the dimension of the spinor representation of $\operatorname{Spin}(N+$ 1) labelled by $n$ (see section 5 ). Of course, the degeneracy of the eigenvalue $-(n+N / 2)^{2}$ of $\nabla^{2}$ is $2 D_{N}(n)$.

Case 2. $N$ odd $(\geq 3)$. In this case a Dirac spinor on $S^{N}$ is irreducible under $\operatorname{Spin}(N)$ and the dimension of the $\Gamma$-matrices is $2^{(N-1) / 2}$, the same as on $S^{N-1}$. The Dirac operator in the geodesic polar coordinates vielbein (3.4) takes the form

$$
\not \nabla \psi=\left(\partial_{\theta}+\rho \cot \theta\right) \Gamma^{N} \psi+\frac{1}{\sin \theta} \tilde{\nabla} \psi
$$

where $\rho=(N-1) / 2$ and $\tilde{\nabla}=\tilde{\Gamma}^{j} \tilde{\nabla}_{j}$ is the Dirac operator (3.11) on $S^{N-1}$. Since

$$
\Gamma^{N} \tilde{\nabla}+\tilde{\nabla} \Gamma^{N}=0
$$

the iterated Dirac operator is given by

$$
\nabla^{2} \psi=\left(\partial_{\theta}+\rho \cot \theta\right)^{2} \psi+\frac{1}{\sin ^{2} \theta} \tilde{\nabla}^{2} \psi-\frac{\cos \theta}{\sin ^{2} \theta} \Gamma^{N} \tilde{\nabla} \psi
$$

In order to separate variables in the eigenvalue equation (3.16) we observe that although $\tilde{\nabla}$ does not commute with $\nabla^{2}$, the operator $\Gamma^{N} \tilde{\nabla}$ does, as a consequence of (3.47). Thus $\nabla^{2}$ and $\Gamma^{N} \tilde{\nabla}$ can have common eigenfunctions. Furthermore, since $(\tilde{\nabla})^{\dagger}=-\tilde{\nabla}$ and $\left(\Gamma^{N}\right)^{\dagger}=\Gamma^{N}$, the operator $\Gamma^{N} \tilde{\nabla}$ is hermitian (because of (3.47)), and has real eigenvalues.

Suppose that we have solved the equation

$$
\Gamma^{N} \tilde{\nabla} \hat{\chi}_{l m}^{( \pm)}= \pm k \hat{\chi}_{l m}^{( \pm)}
$$


for the spinor $\hat{\chi}$ on $S^{N-1}$. In order to find the possible values of $k$ we apply $\tilde{\nabla}$ to both sides of (3.49) and use (3.47) to find

$$
\tilde{\nabla}^{2} \hat{\chi}_{l m}^{( \pm)}=-k^{2} \hat{\chi}_{l m}^{( \pm)}
$$

Thus $-k^{2}$ are the eigenvalues of the iterated Dirac operator on $S^{N-1}$, i.e.

$$
k=l+\rho, \quad l=0,1, \ldots
$$

Let $\chi_{l m}^{(-)}$satisfy

$$
\tilde{\nabla} \chi_{l m}^{(-)}=-i k \chi_{l m}^{(-)}
$$

Then $\chi_{l m}^{(+)} \equiv \Gamma^{N} \chi_{l m}^{(-)}$is the eigenfunction of $\tilde{\not}$ with eigenvalue $+i k$. These are related to $\hat{\chi}_{l m}^{( \pm)}$by

$$
\begin{aligned}
& \hat{\chi}_{l m}^{(-)}=\frac{1}{\sqrt{2}}\left(1+i \Gamma^{N}\right) \chi_{l m}^{(-)}, \\
& \hat{\chi}_{l m}^{(+)}=\Gamma^{N} \hat{\chi}_{l m}^{(-)} .
\end{aligned}
$$

We can now separate variables in (3.16) by letting

$$
\begin{aligned}
& { }^{(1)} \psi_{n l m}(\theta, \Omega)=\phi_{n l}(\theta) \hat{\chi}_{l m}^{(-)}(\Omega), \\
& { }^{(2)} \psi_{n l m}(\theta, \Omega)=\psi_{n l}(\theta) \hat{\chi}_{l m}^{(+)}(\Omega) .
\end{aligned}
$$

Substituting (3.55) in (3.16) and using (3.48) we find that the scalar functions $\phi_{n l}$ satisfy the same equation (3.20) as in the case of $N$ even. We immediately conclude that $\phi_{n l}$ is given by (3.25), and similarly we find that $\psi_{n l}$ is given by (3.27).

The eigenfunctions of the first-order Dirac operator must be of the form

$$
\psi(\theta, \Omega)=\phi_{n l}(\theta) \hat{\chi}_{l m}^{(-)}(\Omega)+c \psi_{n l}(\theta) \hat{\chi}_{l m}^{(+)}(\Omega)
$$

Then, using (3.29) and (3.30) in (3.46), we find that $\psi$ is an eigenfunction of the firstorder Dirac operator with eigenvalues $\pm i\left(n+\frac{N}{2}\right)$ if we choose $c= \pm i$. The normalization factors are given again by $c_{N}(n l) / \sqrt{2}$. The formula for the dimensionality is the same as that for $N$ even except that the $l=0$ representation of $\operatorname{Spin}(N)$ is $2^{\frac{N-1}{2}}$-dimensional (and there is no factor of 2 we had for $N$ even). Thus, the dimensionality is

$$
D_{N}(n)=\frac{2^{(N-1) / 2}(N+n-1) !}{n !(N-1) !} .
$$

Our method shows how to separate variables in the Dirac equation written in geodesic polar coordinates. The spinor modes of $\nabla^{2}$ on $S^{N}$ are the product of the spherical modes $\phi_{n l}, \psi_{n l}$ times the spinor modes on $S^{N-1}[(3.17)(N$ even) or (3.49) ( $N$ odd)]. But again 
these can be obtained from the spinor modes on $S^{N-2}$, and so on. Therefore we have set up an induction procedure by which the spinor modes on $S^{N}$ can be recursively calculated starting from the spinor modes on the lowest dimensional sphere $S^{2}$.

Note also that our procedure gives an independent proof of the spectrum of the Dirac operator $\not$ by induction. First consider the case $N=2$. Writing $d s_{2}^{2}=d \theta^{2}+\sin ^{2} \theta d \varphi^{2}$, one simply has $\tilde{\nabla}=\partial / \partial \varphi$. We need to find which eigenvalues of this operator are allowed. Spinors on $S^{2}$ should transform as a double-valued spin-1/2 representation of $S O(2)$ under the rotation of zweibein. Now, the loop defined by $0 \leq \varphi<2 \pi$ with $\theta=$ const. in the bundle of frames over $S^{2}$ is homotope to the $2 \pi$-rotation at a point for our system of zweibein. Hence, the spinor field must change sign when it goes around this loop. Thus, we have $\partial / \partial \varphi= \pm i / 2, \pm 3 i / 2, \ldots$ ? The modes are given by letting $\chi_{l m}^{( \pm)}(\Omega)=e^{ \pm i(l+1 / 2) \varphi}$ $(l=0,1, \ldots)$ in $(3.18)$ and $(3.19)$. Then regular solutions of the Dirac equation $\not \nabla \psi=i \lambda \psi$ on $S^{2}$ exist only for $\lambda= \pm(n+1)$ with $n(\geq l)$ being a nonnegative integer. Now, assume that the spectrum of the operator $\tilde{\nabla}$ on $S^{N-1}$ is $\pm i(l+\rho), \rho=(N-1) / 2, l=0,1, \ldots$ Then, whether $N$ is even or odd, regular solutions of the Dirac equation on $S^{N}$ exist only for $\lambda= \pm(n+N / 2)$ with $n(\geq l)$ being a nonnegative integer. This completes the proof by induction that the spectrum of the Dirac operator on $S^{N}$ is indeed given by $\pm i(n+N / 2)$ with $n=0,1, \ldots$

\section{$3.2 \quad H^{N}$}

The real hyperbolic space $H^{N}$ is the noncompact partner of $S^{N}$. The metric on $H^{N}$ in geodesic polar coordinates takes the form (3.1) with $\theta \rightarrow y$ and $f(y)=\sinh y$, where $y$ is the geodesic distance from the origin. By repeating the same steps as on $S^{N}$ one obtains a hypergeometric equation for the spherical modes $\phi_{\lambda l}(y)$. The spectrum of $\nabla^{2}$ is now continuous and is labelled by the real parameter $\lambda$ (as in (3.16)). The final results on $H^{N}$ and $S^{N}$ are related by analytic continuation in the geodesic distance. More precisely, one expresses the Jacobi polynomials in terms of the hypergeometric function according to (3.31) and then one makes the replacements

$$
\theta \rightarrow i y, \quad n \rightarrow-i \lambda-\frac{N}{2}
$$

in the spinor modes found above on $S^{N}$. The result for the (unnormalized) spherical modes on $H^{N}$ is

$$
\phi_{\lambda l}(y)=\left(\cosh \frac{y}{2}\right)^{l+1}\left(\sinh \frac{y}{2}\right)^{l} F\left(\frac{N}{2}+l+i \lambda, \frac{N}{2}+l-i \lambda, \frac{N}{2}+l,-\sinh ^{2} \frac{y}{2}\right) .
$$

\footnotetext{
${ }^{2}$ It also follows that the spinor must vanish both at $\theta=0$ and $\pi$ if $\partial / \partial \varphi \neq \pm i / 2$. This is automatically satisfied by requiring regularity of the solutions.
} 
For the modes $\psi_{\lambda l}$ we find similarly

$$
\psi_{\lambda l}(y)=\frac{2 \lambda}{N+2 l}\left(\cosh \frac{y}{2}\right)^{l}\left(\sinh \frac{y}{2}\right)^{l+1} F\left(\frac{N}{2}+l+i \lambda, \frac{N}{2}+l-i \lambda, \frac{N}{2}+l+1,-\sinh ^{2} \frac{y}{2}\right) .
$$

Here $\lambda \in \mathbf{R}$, and $l=0,1, \ldots, \infty$. The eigenfunctions of the first-order Dirac operator can also be obtained by letting $\theta \rightarrow i y$ and $n \rightarrow-i \lambda-\frac{N}{2}$ in (3.32)-(3.33) ( $N$ even) and in $(3.57)\left(N\right.$ odd). Notice from $(3.10)$ and $(3.46)$ that $\left.\left.\nabla\right|_{S^{N}} \rightarrow \frac{1}{i} \nabla\right|_{H^{N}}$ for $\theta \rightarrow i y$. It follows from (3.34) that the solution of the Dirac equation on $H^{N}$ with eigenvalue $i \lambda$ is obtained from that on $S^{N}$ with eigenvalue $i(n+N / 2)$. Thus we find for $N$ even

$$
\not \nabla \psi_{ \pm \lambda l m}^{(-)}= \pm i \lambda \psi_{ \pm \lambda l m}^{(-)}
$$

where

$$
\psi_{ \pm \lambda l m}^{(-)}(y, \Omega) \equiv \frac{c_{N}(\lambda l)}{\sqrt{2}}\left(\begin{array}{c}
\phi_{\lambda l}(y) \chi_{l m}^{(-)}(\Omega) \\
\pm i \psi_{\lambda l}(y) \chi_{l m}^{(-)}(\Omega)
\end{array}\right)
$$

where $c_{N}(\lambda l)$ is a normalization constant. The modes $\psi_{ \pm \lambda l m}^{(+)}$are obtained by interchanging $\phi_{\lambda l}(y)$ and $\psi_{\lambda l}(y)$, and letting $\chi_{l m}^{(-)} \rightarrow \chi_{l m}^{(+)}$on the right-hand side. The asymptotic behavior for large $y$ is obtained as in the scalar case (see, e.g. [4]) as

$$
\begin{aligned}
& \phi_{\lambda l}(y) \sim c_{l}(\lambda) e^{(-\rho+i \lambda) y}+\text { c.c. } \\
& \psi_{\lambda l}(y) \sim i\left[c_{l}(\lambda) e^{(-\rho+i \lambda) y}-\text { c.c. }\right]
\end{aligned}
$$

where

$$
c_{l}(\lambda)=\frac{2^{N-2}}{\sqrt{\pi}} \frac{\Gamma(N / 2+l) \Gamma(1 / 2+i \lambda)}{\Gamma(N / 2+l+i \lambda)} .
$$

Then the (continuous-spectrum) normalization constant can be found, again, with the method used for scalar and tensor fields 4 as

$$
\left|c_{N}(\lambda l)\right|^{2}=c_{N}\left|c_{l}(\lambda)\right|^{-2}
$$

where $c_{N}=2^{N-2} / \pi$. We define the spectral function $\mu(\lambda)$, which is needed to find the heat kernel, by

$$
\mu(\lambda) \equiv \frac{\Omega_{N-1}}{c_{N} g(1 / 2)} \sum_{s l m} \psi_{+\lambda l m}^{(s)}(0)^{\dagger} \psi_{+\lambda l m}^{(s)}(0),
$$

where again $s= \pm$ and the spin factor $g\left(\frac{1}{2}\right)=2^{N / 2}$ is the dimension of the spinor. Then we find

$$
\mu(\lambda)=\left|c_{0}(\lambda)\right|^{-2}=\frac{\pi}{2^{2 N-4}}\left|\frac{\Gamma(N / 2+i \lambda)}{\Gamma(N / 2) \Gamma(1 / 2+i \lambda)}\right|^{2} .
$$

The computation proceeds almost in the same way for $N$ odd and we obtain the same formula for $\mu(\lambda)$. More explicitly the spectral function is given by

$$
\mu(\lambda)=\frac{\pi}{2^{2 N-4} \Gamma(N / 2)^{2}}\left\{\begin{array}{l}
\prod_{j=1 / 2}^{(N-2) / 2}\left(\lambda^{2}+j^{2}\right), \quad N \text { odd }, \\
\lambda \operatorname{coth}(\pi \lambda) \prod_{j=1}^{(N-2) / 2}\left(\lambda^{2}+j^{2}\right), \quad N \text { even } .
\end{array}\right.
$$




\section{The spinor heat kernel}

\subsection{The compact case}

The spinor heat kernel $K\left(x, x^{\prime}, t\right)$ on $S^{N}$ satisfies the heat equation for $\not^{2}$

$$
\left(-\frac{\partial}{\partial t}+\nabla_{x}^{2}\right) K\left(x, x^{\prime}, t\right)=0
$$

with the initial condition

$$
\lim _{t \rightarrow 0} \int_{S^{N}} K\left(x, x^{\prime}, t\right) \psi\left(x^{\prime}\right) d x^{\prime}=\psi(x) .
$$

Here $t$ is the time parameter in the heat equation and we are suppressing all spinor indices. $K$ is actually a $2^{\left[\frac{N}{2}\right]} \times 2^{\left[\frac{N}{2}\right]}$ matrix. $K\left(x, x^{\prime}, t\right)$ maps a spinor at $x^{\prime}$ to a spinor at $x$.

Case 1. $N$ odd. Consider the normalized eigenfunctions of $\not \nabla$ on $S^{N}$ (cf. (3.57))

$$
\psi_{n l m}^{( \pm)}(\theta, \Omega)=\frac{c_{N}(n l)}{\sqrt{2}}\left[\phi_{n l}(\theta) \hat{\chi}_{l m}^{(-)}(\Omega) \pm i \psi_{n l}(\theta) \hat{\chi}_{l m}^{(+)}(\Omega)\right] .
$$

Here $n=0,1, \ldots, \infty, l=0,1, \ldots, n$, and $m=1, \ldots, D_{N-1}(l)$.

The heat kernel is given by the familiar mode expansion

$$
\begin{array}{r}
K\left(\theta, \Omega, \theta^{\prime}, \Omega^{\prime}, t\right)=\sum_{n, l, m}\left[\psi_{n l m}^{(-)}(\theta, \Omega) \otimes \psi_{n l m}^{(-)}\left(\theta^{\prime}, \Omega^{\prime}\right)^{*}\right. \\
\left.+\psi_{n l m}^{(+)}(\theta, \Omega) \otimes \psi_{n l m}^{(+)}\left(\theta^{\prime}, \Omega^{\prime}\right)^{*}\right] e^{-t(n+N / 2)^{2}} .
\end{array}
$$

Using (4.3) in (4.4) we obtain

$$
\begin{aligned}
K\left(\theta, \Omega, \theta^{\prime}, \Omega^{\prime}, t\right)= & \sum_{n, l, m}\left|c_{N}(n l)\right|^{2}\left[\phi_{n l}(\theta) \phi_{n l}\left(\theta^{\prime}\right) \hat{\chi}_{l m}^{(-)}(\Omega) \otimes \hat{\chi}_{l m}^{(-)}\left(\Omega^{\prime}\right)^{*}\right. \\
& \left.+\psi_{n l}(\theta) \psi_{n l}\left(\theta^{\prime}\right) \hat{\chi}_{l m}^{(+)}(\Omega) \otimes \hat{\chi}_{l m}^{(+)}\left(\Omega^{\prime}\right)^{*}\right] e^{-t(n+N / 2)^{2}}
\end{aligned}
$$

The same result would have been obtained by expanding $K$ directly in terms of the (normalized) eigenfunctions (3.55)-(3.56).

In order to simplify the above expression for $K$ we let $\Omega=\Omega^{\prime}$, i.e., we assume that the two points $x=(\theta, \Omega)$ and $x^{\prime}=\left(\theta^{\prime}, \Omega^{\prime}\right)$ lie on the same "meridian". There is no loss of generality in doing so. Then the sum over $m$ can be performed explicitly by using

$$
\sum_{m} \hat{\chi}_{l m}^{(-)}(\Omega) \otimes \hat{\chi}_{l m}^{(-)}(\Omega)^{*}=\sum_{m} \hat{\chi}_{l m}^{(+)}(\Omega) \otimes \hat{\chi}_{l m}^{(+)}(\Omega)^{*}=\frac{D_{N-1}(l)}{D_{N-1}(0) \Omega_{N-1}} \mathbf{1}
$$


This equation can be proved by induction in $N$. Thus

$$
K\left(\theta, \Omega, \theta^{\prime}, \Omega, t\right)=\mathbf{1} \sum_{n, l} \frac{D_{N-1}(l)\left|c_{N}(n l)\right|^{2}}{D_{N-1}(0) \Omega_{N-1}}\left[\phi_{n l}(\theta) \phi_{n l}\left(\theta^{\prime}\right)+\psi_{n l}(\theta) \psi_{n l}\left(\theta^{\prime}\right)\right] e^{-t(n+N / 2)^{2}} .
$$

Notice that the unit matrix $\mathbf{1}$ is just the parallel spinor propagator $U\left(x, x^{\prime}\right)$ between $x^{\prime}$ and $x$. [This is the operator that parallel transports a spinor from $x^{\prime}$ to $x$ along the shortest geodesic (great circle) between them. We assume here that $x$ and $x^{\prime}$ are not conjugate.] Indeed the polar frame (3.4) is parallel along the meridian $\Omega=\Omega^{\prime}=$ constant, and therefore parallel propagation along this curve is trivial in this frame. We rewrite (4.7) as

$$
K\left(\theta, \Omega, \theta^{\prime}, \Omega, t\right)=U\left(x, x^{\prime}\right) f_{N}\left(\theta, \theta^{\prime}, t\right),
$$

where $f_{N}\left(\theta, \theta^{\prime}, t\right)$ denotes the scalar sum over $n, l$ in the right-hand side of (4.7). Notice that any dependence on $\Omega$ has dropped out in $f_{N}$. Therefore, we can let $\theta=0$, i.e, we can assume that $x$ coincides with the north-pole. Recalling that $\psi_{n l}(0)=0 \forall n, l$, and $\phi_{n l}(0)=0 \quad \forall l>0$, we see that only the term with $l=0$ survives in the sum. Thus we get (writing $\theta$ in place of $\theta^{\prime}$ )

$$
f_{N}(\theta, t) \equiv f_{N}(0, \theta, t)=\sum_{n=0}^{\infty} \frac{\left|c_{N}(n 0)\right|^{2}}{\Omega_{N-1}} \phi_{n 0}(0) \phi_{n 0}(\theta) e^{-t(n+N / 2)^{2}}
$$

Using (set $x=1$ in eq. (3.31))

$$
P_{n}^{(\alpha, \beta)}(1)=\frac{\Gamma(n+\alpha+1)}{n ! \Gamma(\alpha+1)},
$$

we get from $(3.25)$

$$
\phi_{n 0}(0)=\frac{\Gamma(n+N / 2)}{n ! \Gamma(N / 2)} .
$$

Recalling (3.38) and using (3.45) we obtain

$$
f_{N}(\theta, t)=\frac{1}{\Omega_{N}} \sum_{n=0}^{\infty} d_{n} \phi_{n}(\theta) e^{-t(n+N / 2)^{2}},
$$

where the $\phi_{n}(\theta)$ are the functions $\phi_{n 0}(\theta)$ normalized by $\phi_{n}(0)=1$,

$$
\phi_{n}(\theta)=\frac{\phi_{n 0}(\theta)}{\phi_{n 0}(0)}=\frac{n ! \Gamma\left(\frac{N}{2}\right)}{\Gamma\left(n+\frac{N}{2}\right)} \cos \frac{\theta}{2} P_{n}^{\left(\frac{N}{2}-1, \frac{N}{2}\right)}(\cos \theta)
$$

and $d_{n}$ are the degeneracies of $\not^{2}$ on $S^{N}$ without the spin factor $2^{\left[\frac{N}{2}\right]}$ :

$$
d_{n}=\frac{2(n+N-1) !}{n !(N-1) !} .
$$


Eqs. (4.12), 4.13) and (4.14) coincide with eqs. (3.22), (3.19), and (3.21) of ref. [3].

The spinor heat kernel on $S^{N}, N$ odd, may finally be written as

$$
K\left(x, x^{\prime}, t\right)=U\left(x, x^{\prime}\right) f_{N}\left(d\left(x, x^{\prime}\right), t\right)
$$

where $d\left(x, x^{\prime}\right)$ denotes the geodesic distance between $x$ and $x^{\prime}$. This form of $K$ is now valid for $x$ and $x^{\prime}$ arbitrary (nonconjugate) points on $S^{N}$. If $x$ and $x^{\prime}$ are conjugate (e.g., $x$ is the north-pole and $x^{\prime}$ the south-pole), the parallel propagator $U\left(x, x^{\prime}\right)$ is clearly undefined (there are infinitely many great semicircles connecting $x^{\prime}$ to $x$ and $U$ depends on the one we choose to parallel propagate). However for conjugate points $d\left(x, x^{\prime}\right)=\pi$ and the heat kernel (4.15) is well defined and vanishes ( since $f_{N}(\pi, t)=0$ ).

Case 2. $N$ even. Consider the normalized eigenfunctions of $\not$ on $S^{N}$ given by (3.32) and (3.33). The heat kernel of $\not^{2}$ is given by the mode expansion

$$
\begin{array}{r}
K\left(\theta, \Omega, \theta^{\prime}, \Omega^{\prime}, t\right)=\sum_{n, l, m}\left[\psi_{-n l m}^{(-)}(\theta, \Omega) \otimes \psi_{-n l m}^{(-)}\left(\theta^{\prime}, \Omega^{\prime}\right)^{*}+\psi_{+n l m}^{(-)}(\theta, \Omega) \otimes \psi_{+n l m}^{(-)}\left(\theta^{\prime}, \Omega^{\prime}\right)^{*}\right. \\
\left.+\psi_{-n l m}^{(+)}(\theta, \Omega) \otimes \psi_{-n l m}^{(+)}\left(\theta^{\prime}, \Omega^{\prime}\right)^{*}+\psi_{+n l m}^{(+)}(\theta, \Omega) \otimes \psi_{+n l m}^{(+)}\left(\theta^{\prime}, \Omega^{\prime}\right)^{*}\right] e^{-t(n+N / 2)^{2}} .
\end{array}
$$

Substituting (3.32)-(3.33) in (4.16) we obtain

$$
K\left(\theta, \Omega, \theta^{\prime}, \Omega^{\prime}, t\right)=\sum_{n, l, m}\left|c_{N}(n l)\right|^{2}\left(\begin{array}{cc}
K_{n l m}^{+} & 0 \\
0 & K_{n l m}^{-}
\end{array}\right) e^{-t(n+N / 2)^{2}},
$$

where

$$
\begin{aligned}
K_{n l m}^{+}\left(\theta, \Omega, \theta^{\prime}, \Omega^{\prime}\right) & =\phi_{n l}(\theta) \phi_{n l}\left(\theta^{\prime}\right) \chi_{l m}^{(-)}(\Omega) \otimes \chi_{l m}^{(-)}\left(\Omega^{\prime}\right)^{*} \\
& +\psi_{n l}(\theta) \psi_{n l}\left(\theta^{\prime}\right) \chi_{l m}^{(+)}(\Omega) \otimes \chi_{l m}^{(+)}\left(\Omega^{\prime}\right)^{*}
\end{aligned}
$$

and

$$
\begin{aligned}
K_{n l m}^{-}\left(\theta, \Omega, \theta^{\prime}, \Omega^{\prime}\right) & =\phi_{n l}(\theta) \phi_{n l}\left(\theta^{\prime}\right) \chi_{l m}^{(+)}(\Omega) \otimes \chi_{l m}^{(+)}\left(\Omega^{\prime}\right)^{*} \\
& +\psi_{n l}(\theta) \psi_{n l}\left(\theta^{\prime}\right) \chi_{l m}^{(-)}(\Omega) \otimes \chi_{l m}^{(-)}\left(\Omega^{\prime}\right)^{*}
\end{aligned}
$$

Note that $K=K^{+} \oplus K^{-}$, where $K^{ \pm}$are the heat kernels for "upper" spinors $\psi^{+} \in \Gamma\left(E^{\tau_{+}}\right)$ and "lower" spinors $\psi^{-} \in \Gamma\left(E^{\tau_{-}}\right)$, respectively (see section 5 for the notations).

Letting, as before, $\Omega=\Omega^{\prime}$, we can do the sum over $m$ in (4.17) as eq. (4.6) is still valid (with $\hat{\chi}^{( \pm)} \rightarrow \chi^{( \pm)}$). The result is

$$
K\left(\theta, \Omega, \theta^{\prime}, \Omega, t\right)=\left(\begin{array}{ll}
\mathbf{1} & 0 \\
0 & \mathbf{1}
\end{array}\right) f_{N}\left(\theta, \theta^{\prime}, t\right),
$$


where $f_{N}\left(\theta, \theta^{\prime}, t\right)$ denotes the sum over $n, l$ in the right-hand side of (4.7).

Again $\operatorname{diag}(\mathbf{1}, \mathbf{1})=U\left(x, x^{\prime}\right)$, the parallel spinor propagator in the polar frame. Since our manipulations of $f_{N}\left(\theta, \theta^{\prime}, t\right)$ were independent of $N$ being odd or even, we obtain, by letting $\theta \rightarrow 0, f_{N}(0, \theta, t)=f_{N}(\theta, t)$ given by (4.12). Finally the spinor heat kernel on $S^{N}, N$ even, takes the same form (4.15).

\subsection{The noncompact case}

Case 1. $N$ odd. The normalized eigenfunctions of $\not \nabla$ on $H^{N}$ are (cf. (4.3))

$$
\psi_{\lambda l m}^{( \pm)}(y, \Omega)=\frac{c_{N}(\lambda l)}{\sqrt{2}}\left[\phi_{\lambda l}(y) \hat{\chi}_{l m}^{(-)}(\Omega) \pm i \psi_{\lambda l}(y) \hat{\chi}_{l m}^{(+)}(\Omega)\right],
$$

where $\lambda$ is a real number and may be taken to be positive, $l=0,1, \ldots, \infty$, and $m=$ $1, \ldots, D_{N-1}(l)$. The functions $\phi_{\lambda l}, \psi_{\lambda l}$, and the normalization factor $c_{N}(\lambda l)$ are given by eqs. (3.60), (3.61), and (3.67), respectively. The modes (4.21) are eigenfunctions of $\nabla$ with eigenvalues $\pm i \lambda$ (cf. (3.62)). They satisfy the continuous spectrum normalization

$$
\int_{0}^{+\infty} \int_{S^{N-1}} \psi_{\lambda l m}^{(s)}(y, \Omega)^{\dagger} \psi_{\lambda^{\prime} l^{\prime} m^{\prime}}^{\left(s^{\prime}\right)}(y, \Omega)(\sinh y)^{N-1} d y d \Omega_{N-1}=\delta\left(\lambda-\lambda^{\prime}\right) \delta_{l l^{\prime}} \delta_{m m^{\prime}} \delta_{s s^{\prime}}
$$

where $s, s^{\prime}= \pm$ and $\delta\left(\lambda-\lambda^{\prime}\right)$ is the Dirac distribution. The heat kernel of $\not^{2}$ is given by

$$
\begin{array}{r}
K\left(y, \Omega, y^{\prime}, \Omega^{\prime}, t\right)=\int_{0}^{+\infty} \sum_{l, m}\left[\psi_{\lambda l m}^{(-)}(y, \Omega) \otimes \psi_{\lambda l m}^{(-)}\left(y^{\prime}, \Omega^{\prime}\right)^{*}+\right. \\
\left.\psi_{\lambda l m}^{(+)}(y, \Omega) \otimes \psi_{\lambda l m}^{(+)}\left(y^{\prime}, \Omega^{\prime}\right)^{*}\right] e^{-t \lambda^{2}} d \lambda \\
=\int_{0}^{+\infty} \sum_{l, m}\left|c_{N}(\lambda l)\right|^{2}\left[\phi_{\lambda l}(y) \phi_{\lambda l}\left(y^{\prime}\right) \hat{\chi}_{l m}^{(-)}(\Omega) \otimes \hat{\chi}_{l m}^{(-)}\left(\Omega^{\prime}\right)^{*}+\right. \\
\left.\psi_{\lambda l}(y) \psi_{\lambda l}\left(y^{\prime}\right) \hat{\chi}_{l m}^{(+)}(\Omega) \otimes \hat{\chi}_{l m}^{(+)}\left(\Omega^{\prime}\right)^{*}\right] e^{-t \lambda^{2}} .
\end{array}
$$

We now proceed like in the case of $S^{N}$. We set $\Omega=\Omega^{\prime}$ and do the sum over $m$ using (4.6). Then we let $y=0$, i.e. we fix the point $x$ at the origin. The final result, for arbitrary points $x, x^{\prime} \in H^{N}$, is

$$
\begin{gathered}
K\left(x, x^{\prime}, t\right)=U\left(x, x^{\prime}\right) \hat{f}_{N}\left(d\left(x, x^{\prime}\right), t\right), \\
\hat{f}_{N}(y, t)=\frac{2^{N-3} \Gamma(N / 2)}{\pi^{N / 2+1}} \int_{0}^{+\infty} \phi_{\lambda}(y) e^{-t \lambda^{2}} \mu(\lambda) d \lambda,
\end{gathered}
$$

where $\phi_{\lambda}=\phi_{\lambda 0}, \mu(\lambda)$ is the spinor spectral function (3.69), and $U$ is the parallel propagator. This agrees with eq. (5.14) of ref. [3].

Case 2. $N$ even. The normalized eigenfunctions of $\not \nabla$ are given by (3.63). In terms of these modes the heat kernel is given by a formula similar to (4.16), with $\sum_{n} \rightarrow \int_{0}^{\infty} d \lambda$. The computation proceeds in the same way as for $N$ odd and we obtain for $K$ the same expression (4.25)-(4.26), again in agreement with ref. [3]. 


\section{Group theoretic derivation of the heat kernel}

In this section we give a group-theoretic derivation of the spectrum, the eigenfunctions, and the heat kernel of the iterated Dirac operator on $S^{N}$ and $H^{N}$. We begin by reviewing some basic facts about harmonic analysis for homogeneous vector bundles over compact symmetric spaces (SS) (see, e.g. [2] appendix B).

\subsection{Harmonic analysis on compact symmetric spaces}

Let $U$ be a compact semisimple Lie group and $K$ a closed subgroup such that the coset space $U / K$ is diffeomorphic to a Riemannian symmetric space of the compact type. Let $x \rightarrow u x(u \in U, x \in U / K)$ be the natural left-action of $U$ on $U / K$, and let $\pi$ denote the projection of $U$ onto $U / K, \pi(u)=u K$.

The coset $x_{0}=e K$ is called the origin of $U / K$. Let $\sigma: \mathcal{O} \subset U / K \rightarrow U$ be a local section of the principal bundle $U(U / K, K)$ (see e.g. ref. [13]), i.e. a smooth assignment of an element $\sigma(x) \in U$ to any $x$ in an open neighborhood $\mathcal{O}$ of $x_{0}$ such that $\pi(\sigma(x))=x$. Then $\sigma(x) x_{0}=x$. Let $\mathcal{U}$ and $\mathcal{K}$ be the Lie algebras of $U$ and $K$, respectively, and let $A d(U)$ denote the adjoint representation of $U$ in $\mathcal{U}$. Since $K$ is compact, $U / K$ is a reductive coset space [15], i.e. we have a direct sum decomposition $\mathcal{U}=\mathcal{K} \oplus \mathcal{P}$, where $\mathcal{P}$ is a complementary $A d(K)$-invariant subspace of $\mathcal{U}$. $\mathcal{P}$ may be identified with the tangent space $T_{x_{0}}(U / K)$. The natural choice for $\mathcal{P}$ (that will be made in the following) is $\mathcal{P}=\mathcal{K}^{\perp}$, the orthogonal complement of $\mathcal{K}$ in $\mathcal{U}$ with respect to the Killing form. We shall assume that a $U$-invariant metric has been fixed on $U / K$ by restricting the opposite of the Killing form (or a multiple of it) from $\mathcal{U}$ to $\mathcal{P}$.

Let Exp and exp denote the exponential mappings on $U / K$ and $U$, respectively. For a symmetric space it is well known (see [9]) that $\operatorname{Exp} X=\pi(\exp X), X \in \mathcal{P}$. Thus on a SS a natural choice for $\sigma$ is

$$
\sigma(\operatorname{Exp} X)=\exp X, \quad X \in \mathcal{P}_{0}
$$

or equivalently $\sigma\left(\exp X x_{0}\right)=\exp X$, where $\mathcal{P}_{0} \subset \mathcal{P}$ is the largest open domain of $\mathcal{P}$ containing the origin and such that the exponential map (restricted to $\mathcal{P}_{0}$ ) is a diffeomorphism.

Let $\partial \mathcal{P}_{0}$ denote the boundary of $\mathcal{P}_{0}$. Then $\sigma$ maps $U / K \backslash \mathcal{C}_{0}$ onto $\exp \left(\mathcal{P}_{0}\right) \subset U$, where $\mathcal{C}_{0}=\operatorname{Exp}\left(\partial \mathcal{P}_{0}\right)$ is called the cut locus of $x_{0}$. It is known that $\mathcal{C}_{0}$ consists either of points that are conjugate to $x_{0}$, or of points that can be joined to $x_{0}$ by more than one minimizing geodesic (see [13 vol.II p.97 Theorem 7.1). In any case $\mathcal{C}_{0}$ has always zero measure. 
The above choice of local section $\sigma$ will be assumed in the following. Let $\left\{Y_{i}\right\}$ be an orthonormal basis of $\mathcal{P}$. Then the moving frame $\left\{Y_{i}^{*}\right\}$ on $U / K \backslash \mathcal{C}_{0}$ defined by $Y_{i}^{*}(\operatorname{Exp} X)=(\exp X)_{* \mid x_{0}} Y_{i}$ is orthonormal, and it is parallel along each geodesic emanating from the origin (see [10] Theorem 3.3 p.208). This is the local frame we shall work with.

Consider a field $x \rightarrow \boldsymbol{\psi}(x)$ on $U / K$ which is a cross section of the homogeneous vector bundle $E^{\tau}$ on $U / K$ defined by a finite dimensional linear representation $\tau$ of the isotropy group $K$ (see [18 section 5.2). For example scalars, vectors and spinors correspond to the trivial, isotropy, and spinor representations of $K$, respectively. 5 The vector space of all sections of $E^{\tau}$ will be denoted by $\Gamma\left(E^{\tau}\right)$. We shall assume that the representation $\tau$ of $K$ be irreducible of dimension $d_{\tau}$. The representation space of $\tau$, denoted $V_{\tau}$, will be identified with the typical fibre $E_{x_{0}}$ of $E^{\tau}$ at the origin.

By definition of homogeneous vector bundle (see [18] 5.2.1), the group $U$ acts on $E^{\tau}$ so that the fiber at $x$ is mapped by $u$ isomorphically onto the fiber at $u x$, for each $x \in U / K$ and each $u \in U$. Notice that $\sigma(x) E_{x_{0}}=E_{x}$. The map $\exp X(X \in \mathcal{P})$ between the fibres at $x_{0}$ and $x=\operatorname{Exp} X$ is the same as the map of parallel transport (in the vector bundle) from $x_{0}$ to $x$ along the geodesic $\gamma_{X}(t)=\exp (t X) x_{0}$. In other words, the parallel displacement of any $\mathbf{v} \in E_{x_{0}}$ along $\gamma_{X}(t)$ is defined to be $\exp (t X) \mathbf{v}$. Let $X^{*}$ be the vector field on $U / K \backslash \mathcal{C}_{0}$ defined by $X^{*}(\operatorname{Exp} Y)=(\exp Y)_{* \mid x_{0}} X(X \in \mathcal{P})$. It is then easy to see that the geodesic $\gamma_{X}(t)=\exp (t X) x_{0}$ is the integral curve through the origin of $X^{*}$, i.e. $d \gamma_{X} / d t=X^{*}\left(\gamma_{X}(t)\right)$. The covariant derivative $\nabla_{X^{*}} \boldsymbol{\psi}$ of $\boldsymbol{\psi} \in \Gamma\left(E^{\tau}\right)$ at $x=x_{0}$ is then defined by

$$
\left(\nabla_{X^{*}} \boldsymbol{\psi}\right)\left(x_{0}\right)=\lim _{t \rightarrow 0} \frac{1}{t}\left[\exp (-t X) \boldsymbol{\psi}\left(\gamma_{X}(t)\right)-\boldsymbol{\psi}\left(x_{0}\right)\right] .
$$

When $E^{\tau}$ is the tangent bundle on $U / K$, the $U$-invariant affine connection defined this way is called the canonical connection of the second kind, see [15]. The elements $\exp X$ $(X \in \mathcal{P})$ are called transvections, see [10] p.209.

Let $\left\{\mathbf{v}_{a}\right\}$ be a fixed orthonormal basis of $V_{\tau} \simeq E_{x_{0}}$. Then we choose as basis for the fibre $E_{x}$ at $x=\operatorname{Exp} X\left(X \in \mathcal{P}_{0}\right)$ the set $\left\{\boldsymbol{\theta}_{a}(x)\right\}$ obtained by transforming $\left\{\mathbf{v}_{a}\right\}$ with $\sigma(x)=\exp X$, i.e. $\boldsymbol{\theta}_{a}(x)=\sigma(x) \mathbf{v}_{a}$. The field $\boldsymbol{\psi}$ is written as $\boldsymbol{\psi}(x)=\sum_{a=1}^{d_{\tau}} \psi^{a}(x) \boldsymbol{\theta}_{a}(x)$ for $x \in U / K \backslash \mathcal{C}_{0}$.

The set of equivalence classes of irreducible unitary representations (irreps) of $U$ and $K$ will be denoted by $\hat{U}$ and $\hat{K}$, respectively. A simple application of the Frobenius

\footnotetext{
${ }^{3}$ The spinor representation of $K$ is obtained by lifting to $\operatorname{Spin}(N), N=\operatorname{dim} U / K$, the Lie homomorphism of $K$ into $S O(N)$ induced by the isotropy representation $k \rightarrow k_{* \mid x_{0}}$ by using an orthonormal basis of $T_{x_{0}}(U / K)$. There may be topological obstructions to the (global) existence of spinors. We recall that an oriented Riemannian manifold $M$ admits a spin structure if and only if the second Stiefel-Whitney class of $M$ vanishes.
} 
Reciprocity Theorem to the induced representation of $U$ on $L^{2}\left(U / K, E^{\tau}\right)$ (the space of square-integrable sections of $E^{\tau}$ ) gives the following harmonic expansion for the components $\psi^{a}(x)$ (here $\sigma^{-1}(x) \equiv(\sigma(x))^{-1}$, see [2] appendix B)

$$
\psi^{a}(x)=\sum_{\lambda \in \hat{U}(\tau)} \sum_{I=1}^{d_{\lambda}} \sum_{\xi=1}^{\xi_{\lambda}} \psi_{\lambda \xi}^{I} U^{\lambda}\left(\sigma^{-1}(x)\right)_{I}^{a \xi}, \quad a=1, \ldots, d_{\tau} .
$$

Here $\hat{U}(\tau)$ is the set of irreps $\lambda \in \hat{U}$ (of dimension $d_{\lambda}$ ) such that $\left.\lambda\right|_{K}$ contains $\tau$ at least once. The index $\xi$ labels the multiplicity $\xi_{\lambda}$ of $\tau$ in $\left.\lambda\right|_{K}$, thus $\xi=1, \ldots, \xi_{\lambda}$. Each representation space $V_{\lambda}$ is decomposed as $\left(\oplus_{\xi} V_{\xi}\right) \oplus V_{\text {rest }}$, where $\left.\lambda\right|_{K}$ is equivalent to $\tau$ in each $V_{\xi}$, and $V_{\text {rest }}=\left(\oplus_{\xi} V_{\xi}\right)^{\perp}$. Thus each $V_{\xi}$ is isomorphic to $V_{\tau}$ and $\operatorname{dim} V_{\xi}=$ $d_{\tau} \forall \xi . U^{\lambda}(u)^{I}{ }_{J}(u \in U)$ are the matrix coefficients of the operator $U^{\lambda}(u)$ of the irrep $\lambda$ in a (fixed) orthonormal basis $\left\{\mathbf{v}_{I}\right\}$ of $V_{\lambda}$ adapted to the above decomposition. Thus $U^{\lambda}(u)^{a \xi}{ }_{I}=<U^{\lambda}(u) \mathbf{v}_{I}, \mathbf{v}_{a \xi}>$, where $\left\{\mathbf{v}_{a \xi}\right\}_{a=1, \ldots, d_{\tau}}$ is an orthonormal basis of $V_{\xi}$.

For scalar fields on a SS it is known (e.g. ref. [11]) that each $\lambda \in \hat{U}(\tau)$ (where $\tau$ is the trivial representation of $K$ ) contains $\tau$ exactly once, so that $\xi_{\lambda}=1 \forall \lambda$. For arbitrary fields on arbitrary symmetric spaces $\xi_{\lambda} \geq 1$ and the sum over $\xi$ in (5.3) is generally nontrivial. f In the case of $U=\operatorname{Spin}(N+1), S U(N+1)$ and $K=\operatorname{Spin}(N), S(U(1) \times$ $U(N)$ ), respectively, it can be proved (see e.g. 14]) that any $\tau \in \hat{K}$ appears in any $\lambda \in \hat{U}$ at most once.

Let $d x$ be the invariant measure on $U / K$ (induced by the Riemannian structure) normalized by $\int_{U / K} d x=1$. Then the "Fourier coefficients" $\psi_{\lambda \xi}^{I}$ in (5.3) can be determined by the formula

$$
\psi_{\lambda \xi}^{I}=\frac{d_{\lambda}}{d_{\tau}} \int_{U / K} \sum_{a=1}^{d_{\tau}} \overline{U^{\lambda}\left(\sigma^{-1}(x)\right)^{a \xi} I} \psi^{a}(x) d x,
$$

where we have used the Schur relations for the compact group $U$. The matrix coefficients in eq. (5.3) satisfy the orthogonality condition on $U / K$ :

$$
\int_{U / K} \sum_{a} \overline{U^{\lambda}\left(\sigma^{-1}(x)\right)^{a \xi}} U^{\lambda^{\prime}}\left(\sigma^{-1}(x)\right)^{a \xi^{\prime}}{ }_{I^{\prime}} d x=\frac{d_{\tau}}{d_{\lambda}} \delta_{\lambda \lambda^{\prime}} \delta_{I I^{\prime}} \delta_{\xi \xi^{\prime}}
$$

It is well known that the Levi-Civita connection of any $U$-invariant metric on the symmetric space $U / K$ coincides with the canonical connection of the second kind [15]. It follows from this that the matrix-valued functions on $U / K$ given by $x \rightarrow U^{\lambda}\left(\sigma^{-1}(x)\right)^{a \xi}{ }_{I}$ are eigenfunctions of the vector-bundle Laplacian $L_{U / K}$ with eigenvalues [

$$
-\omega_{\lambda}=C_{2}(\tau)-C_{2}(\lambda)
$$

\footnotetext{
${ }^{4}$ It is known [0, 12] that $\xi_{\lambda}$ is bounded by $d_{\tau}$, giving an estimate independent of $\lambda$. There is a formula of Kostant for the multiplicity of $\tau$ in $\left.\lambda\right|_{K}$, which, however, is too complicated for actual computation.

${ }^{5}$ The Laplacian $L_{U / K}$ is the $U$-invariant differential operator on $L^{2}\left(U / K, E^{\tau}\right)$ induced by the element $\sum_{i} Y_{i}^{2}$ of the universal enveloping algebra of $\mathcal{U}$, where $\left\{Y_{i}\right\}$ is an orthonormal basis of $\mathcal{P}$. It is defined
} 
where $C_{2}(\mu)$ denotes the (second order) Casimir number of an irrep $\mu$. The degeneracy of $-\omega_{\lambda}$ is $d_{\lambda} \xi_{\lambda}$. [We are not considering here the additional degeneracy coming from the fact that different $\lambda$ 's in $\hat{U}(\tau)$ can have the same $C_{2}(\lambda)$. If the sum over $\lambda$ in eq. (5.18) below is meant to be a sum over different eigenvalues, then the true degeneracy of the eigenvalue $-\omega$ is $\sum_{\lambda \in A_{\omega}} d_{\lambda} \xi_{\lambda}$, where $A_{\omega}$ is defined as the set of all $\lambda$ 's in $\hat{U}(\tau)$ with Casimir value $C_{2}(\lambda)=\omega+C_{2}(\tau)$.]

Consider now the heat kernel $K(x, y, t)$ of the vector-bundle Laplacian acting on $L^{2}\left(U / K, E^{\tau}\right)$. For $t \in \mathbf{R}^{+}$and $x, y \in U / K, K(x, y, t)$ is an element of $\operatorname{Hom}\left(E_{y}, E_{x}\right) \simeq$ $E_{x} \otimes E_{y}^{*}$, i.e. a linear map from the fiber at $y$ to the fiber at $x$. [We recall that if $V, W$ are vector spaces we have the isomorphism $\operatorname{Hom}(V, W)=W \otimes V^{*}$, where $V^{*}$ is the dual of $V$, see [19] vol.I p.126.] Let $p_{1}$ and $p_{2}$ be the projections from $U / K \times U / K$ onto the first and second factor $U / K$ respectively. For a vector bundle $E$ on $U / K$ let $p_{1}^{*} E$ and $p_{2}^{*} E$ denote the pull-back of $E$ relative to $p_{1}$ and $p_{2}$ respectively (cf. [18 p.4). Then the heat kernel $K(\cdot, \cdot, t)$ is a section of the bundle $p_{1}^{*} E^{\tau} \otimes p_{2}^{*} E^{\tau *}$ over $U / K \times U / K$, where $E^{\tau *}$ is the dual bundle to $E^{\tau}$. The heat kernel satisfies the differential equation $\left(L=L_{U / K}\right)$

$$
\left(-\frac{\partial}{\partial t}+L_{x}\right) K(x, y, t)=0
$$

with the initial condition

$$
\lim _{t \rightarrow 0^{+}} \int_{U / K} K(x, y, t) \boldsymbol{\psi}(y) d y=\boldsymbol{\psi}(x)
$$

for any continuous section $\boldsymbol{\psi} \in \Gamma\left(E^{\tau}\right)$. We shall denote by $K(x, y, t)^{a}{ }_{b}\left(a, b=1, \ldots, d_{\tau}\right)$ the matrix representing the endomorphism $K(x, y, t) \in \operatorname{Hom}\left(E_{y}, E_{x}\right)$ in the bases $\left\{\boldsymbol{\theta}_{a}(y)\right\}$ and $\left\{\boldsymbol{\theta}_{a}(x)\right\}$ of $E_{y}$ and $E_{x}$ respectively, for $x, y \in U / K \backslash \mathcal{C}_{0}$. From eqs. (5.3), (5.4), (5.5) and (5.6) we obtain the following eigenfunction expansion of $K(x, y, t)^{a}{ }_{b}$ :

$$
\begin{aligned}
& K(x, y, t)^{a}{ }_{b}=\frac{1}{d_{\tau}} \sum_{\lambda, \xi, I} d_{\lambda} U^{\lambda}\left(\sigma^{-1}(x)\right)^{a \xi}{ }_{I} \overline{U^{\lambda}\left(\sigma^{-1}(y)\right)^{b \xi}} e^{-t \omega_{\lambda}} \\
= & \frac{1}{d_{\tau}} \sum_{\lambda \in \hat{U}(\tau)} \sum_{\xi} d_{\lambda} U^{\lambda}\left(\sigma^{-1}(x) \sigma(y)\right)^{a \xi}{ }_{b \xi} e^{-t \omega_{\lambda}}, \quad a, b=1, \ldots, d_{\tau} .
\end{aligned}
$$

The harmonic expansions (5.3) and (5.9) can also be written in index-free notation as follows. For each $\lambda \in \hat{U}(\tau)$ let $P_{\tau}$ be the projector of $V_{\lambda}$ onto $H_{\tau} \equiv \oplus_{\xi} V_{\xi}$, the subspace of vectors of $V_{\lambda}$ which transform under $K$ according to $\tau$. $H_{\tau}$ will be identified with $V_{\tau} \otimes \mathbf{C}^{\xi_{\lambda}}$, where $\xi_{\lambda}$ is the multiplicity of $\tau$ in $\left.\lambda\right|_{K}$. Define the (operator-valued) by $L_{U / K} \boldsymbol{\psi}=\sum_{i} \nabla_{Y_{i}^{*}} \nabla_{Y_{i}^{*}} \boldsymbol{\psi}$ (cf. (5.2)). Eq. (5.6) follows from the relation $L_{U / K}=\Omega_{U}-\Omega_{K}$, where $\Omega_{U}=\sum_{i} T_{i}^{2}$ and $\Omega_{K}=\sum_{i} X_{i}^{2}$ are the Casimir elements of $U$ and $K\left(\left\{T_{i}\right\}\right.$ and $\left\{X_{i}\right\}$ are orthonormal bases of $\mathcal{U}$ and $\mathcal{K}$, respectively). 
$\tau$-spherical functions $u \rightarrow \Phi_{\tau}^{\lambda}(u)$ on $U$ by

$$
\Phi_{\tau}^{\lambda}(u)=P_{\tau} U^{\lambda}(u) P_{\tau}
$$

Each $\Phi_{\tau}^{\lambda}(u)(u \in U)$ is regarded as a linear operator on $H_{\tau}$. Let $\left\{\mathbf{v}_{a}\right\}$ and $\left\{\mathbf{e}_{\xi}\right\}$ be (fixed) orthonormal bases of $V_{\tau}$ and $\mathbf{C}^{\xi_{\lambda}}$, respectively. Then $\left\{\mathbf{v}_{a \xi}\right\} \equiv\left\{\mathbf{v}_{a} \otimes \mathbf{e}_{\xi}\right\}$ is an orthonormal basis of $H_{\tau}$, and the matrix-valued function on $U$ given by

$$
u \rightarrow \Phi_{\tau}^{\lambda}(u)^{a \xi}{ }_{b \xi^{\prime}} \equiv U^{\lambda}(u)^{a \xi}{ }_{b \xi^{\prime}}, \quad \lambda \in \hat{U}(\tau), \quad \xi, \xi^{\prime}=1, \ldots, \xi_{\lambda}, \quad a, b=1, \ldots, d_{\tau}
$$

(in the notations of eq. (5.3)) is just the matrix representing $\Phi_{\tau}^{\lambda}(u)$ in the basis $\left\{\mathbf{v}_{a \xi}\right\}$.

Let $\varphi_{\tau}^{\lambda}(u)$ denote the partial trace of $\Phi_{\tau}^{\lambda}(u)$ with respect to $\mathbf{C}^{\xi_{\lambda}}$, i.e. in matrix notation

$$
\varphi_{\tau}^{\lambda}(u)^{a}{ }_{b}=\sum_{\xi=1}^{\xi_{\lambda}} \Phi_{\tau}^{\lambda}(u)^{a \xi}{ }_{b \xi} .
$$

The operator $\varphi_{\tau}^{\lambda}(u)$ is regarded as an element of $\operatorname{Hom}\left(V_{\tau}, V_{\tau}\right)$ and can be expressed as

$$
\varphi_{\tau}^{\lambda}(u)=d_{\tau} \int_{K} \phi_{\tau}^{\lambda}\left(u k^{-1}\right) \tau(k) d k
$$

where $\phi_{\tau}^{\lambda}(u)=\operatorname{Tr} \Phi_{\tau}^{\lambda}(u)$ is known as spherical trace function of type $\tau$.

For $x, y \in U / K$ write $x=u x_{0}, y=v x_{0}(u, v \in U)$, and define $\varphi_{\lambda}(x, y) \in \operatorname{Hom}\left(E_{y}, E_{x}\right)$ by

$$
\varphi_{\lambda}(x, y)=u \varphi_{\tau}^{\lambda}\left(u^{-1} v\right) v^{-1}
$$

Here $v^{-1} \in \operatorname{Hom}\left(E_{y}, E_{x_{0}}\right)$ and $u \in \operatorname{Hom}\left(E_{x_{0}}, E_{x}\right)$ are the linear maps guaranteed by the definition of homogeneous vector bundle. It is easy to see that $\varphi_{\lambda}(x, y)$ is well defined, i.e. that the right hand side of (5.14) is invariant under $u \rightarrow u k$ and $v \rightarrow v k^{\prime}\left(k, k^{\prime} \in K\right)$.

Eqs. (5.3) and (5.9) can then be rewritten in operator notation as

$$
\begin{gathered}
\boldsymbol{\psi}(x)=\frac{1}{d_{\tau}} \sum_{\lambda \in \hat{U}(\tau)} d_{\lambda} \int_{U / K} \varphi_{\lambda}(x, y) \boldsymbol{\psi}(y) d y \\
K(x, y, t)=\frac{1}{d_{\tau}} \sum_{\lambda \in \hat{U}(\tau)} d_{\lambda} \varphi_{\lambda}(x, y) e^{-t \omega_{\lambda}} .
\end{gathered}
$$

Eq. (5.16) shows clearly that the heat kernel $K(x, y, t)$ is well defined for all $x$ and $y$ in $U / K$. Moreover we immediately find for the adjoints $\varphi_{\lambda}(x, y)^{\dagger}=\varphi_{\lambda}(y, x)$, and similarly $K(x, y, t)^{\dagger}=K(y, x, t)$. By choosing $u=\sigma(x)$ and $v=\sigma(y)$ in (5.14) $\left(x, y \in U / K \backslash \mathcal{C}_{0}\right)$ we can express $\varphi_{\lambda}(x, y)$ in geometric form as

$$
\varphi_{\lambda}(x, y)=U\left(x, x_{0}\right) \varphi_{\tau}^{\lambda}\left(\sigma^{-1}(x) \sigma(y)\right) U\left(x_{0}, y\right)
$$


where $U\left(x, x_{0}\right)$ (resp. $\left.U\left(x_{0}, y\right)\right)$ is the (vector-bundle) parallel transport operator from $x_{0}$ to $x$ (resp. from $y$ to $x_{0}$ ) along the shortest geodesic between them.

In particular for $x=y$ we have $\varphi_{\lambda}(x, x)=\mathbf{1}$ (the identity operator on $E_{x}$ ), and

$$
K(x, x, t)=\mathbf{1} \frac{1}{d_{\tau}} \sum_{\lambda} d_{\lambda} \xi_{\lambda} e^{-t \omega_{\lambda}}
$$

Taking the trace of this equation and integrating over the manifold gives the partition function.

Suppose now that we fix one of the two points, say $x$, at the origin $x_{0}$ of $U / K$. Then

$$
K(x, t)^{a}{ }_{b} \equiv K\left(x_{0}, x, t\right)^{a}{ }_{b}=\frac{1}{d_{\tau}} \sum_{\lambda, \xi} d_{\lambda} U^{\lambda}(\sigma(x))^{a \xi}{ }_{b \xi} e^{-t \omega_{\lambda}}
$$

or, in operator notation, (cf. (5.16)-(5.17))

$$
K(x, t) \equiv K\left(x_{0}, x, t\right)=\frac{1}{d_{\tau}} \sum_{\lambda} d_{\lambda} \varphi_{\tau}^{\lambda}(\sigma(x)) U\left(x_{0}, x\right) e^{-t \omega_{\lambda}} .
$$

It is easy to find the transformation property of $K(x, t)^{a}{ }_{b}$ under the action of $K$. If $x=\operatorname{Exp} X\left(X \in \mathcal{P}_{0}\right)$, it is easy to see that $k x=\operatorname{Exp}[\operatorname{Ad}(k) X](k \in K)$. Then

$$
\sigma(k x)=\exp [A d(k) X]=k \exp (X) k^{-1}, \quad k \in K
$$

From this and (5.19) we find

$$
K(k x, t)^{a}{ }_{b}=\sum_{c, d} \tau(k)^{a}{ }_{c} K(x, t)^{c}{ }_{d} \tau\left(k^{-1}\right)^{d}{ }_{b}
$$

The trace

$$
\operatorname{Tr}\left[K\left(x_{0}, x, t\right) U\left(x, x_{0}\right)\right]=\sum_{a} K(x, t)^{a}{ }_{a}=\frac{1}{d_{\tau}} \sum_{\lambda} d_{\lambda} \phi_{\tau}^{\lambda}(\sigma(x)) e^{-t \omega_{\lambda}}
$$

is clearly invariant under the action of $K$ (it is a zonal function), thus it can only depend on the coordinates along a submanifold orthogonal to the orbits of $K$. For a symmetric space $U / K$ these submanifolds are maximal tori. Let $\mathcal{A}$ be a (fixed) maximal abelian subspace of $\mathcal{P}$. Then the set $A=\exp \mathcal{A}$ is a torus in $U$, and $\operatorname{Exp} \mathcal{A}=A x_{0}$ is a maximal torus in $U / K$, i.e. a maximal, totally geodesic, flat submanifold passing through the origin. The dimension $l$ of $A$ is called the rank of $U / K$. The maximal tori are all conjugate under the action of $K$ and intersect the orbits of $K$ orthogonally. In the socalled "polar coordinate" decomposition, every $x$ in $U / K$ can be written (nonuniquely) as $x=k a x_{0}$, with $k \in K$ and $a \in A$. More precisely, let $M$ be the centralizer of $A$ in $K$, i.e. the set of those elements $m$ of $K$ such that $m a=a m$ for each $a \in A$. This is a closed subgroup of $K$, and the coset space $K / M$ is diffeomorphic to the orbits of 
$K$ in $U / K$. Clearly $\operatorname{kmax}_{0}=k a x_{0}$, and we can define a map $\Psi: K / M \times A \rightarrow U / K$, by $\Psi(k M, a)=k a x_{0}$. The properties of this map are discussed e.g. in 11] p.188. To illustrate the main ideas, let us suppose that $U$ is simply connected and $K$ is connected, so that $U / K$ is simply connected. Then there is an open set $\mathcal{Q}_{0}$ in $\mathcal{A}$, whose closure $\overline{\mathcal{Q}}_{0}$ contains the origin, such that the mapping

$$
\Psi:(k M, h) \rightarrow k(\exp h) x_{0}
$$

maps $K / M \times \overline{\mathcal{Q}}_{0}$ onto $U / K$ and gives a bijection of $K / M \times \mathcal{Q}_{0}$ onto a certain subset $(U / K)_{r}$ of $U / K$ whose complement has zero measure (see [11] Th.5.11 p.190). f

Let $d u, d k, d m$, and $d k_{M}$ be the invariant measures on $U, K, M$, and $K / M$ normalized by

$$
\int_{U} d u=\int_{K} d k=\int_{M} d m=\int_{K / M} d k_{M}=1
$$

Let $d h$ be the Euclidean measure on $\mathcal{A}$ induced by the scalar product in $\mathcal{P}$. Then we have the integral formula

$$
\int_{U / K} f(x) d x=c \int_{K / M} \int_{\mathcal{Q}_{0}} f\left(k(\exp h) x_{0}\right) J^{2}(h) d h d k_{M}
$$

where the function $J^{2}(h)\left(h \in \mathcal{Q}_{0}\right)$ is given in terms of the positive restricted roots $\alpha$ of the symmetric space (with multiplicity $m_{\alpha}$ ) by

$$
J^{2}(h)=\prod_{\alpha>0}\left(\sin (\alpha(h))^{m_{\alpha}}\right.
$$

and the constant $c$ is determined by

$$
c^{-1}=\int_{\mathcal{Q}_{0}} J^{2}(h) d h .
$$

We also have a polar decomposition for $U$, namely every $u \in U$ can be written (nonuniquely) as $k_{1} a k_{2}$, with $k_{1}, k_{2} \in K$ and $a \in A$. Using (5.26) and the general coset space formulas $\left(d u_{K} \equiv d x\right)$

$$
\begin{gathered}
\int_{U} f(u) d u=\int_{U / K}\left(\int_{K} f(u k) d k\right) d u_{K}, \quad f \in C(U), \\
\int_{K} f(k) d k=\int_{K / M}\left(\int_{M} f(k m) d m\right) d k_{M}, \quad f \in C(K),
\end{gathered}
$$

${ }^{6}$ The set $\mathcal{Q}_{0}$ is any component of $\mathcal{A}_{r}$ whose closure contains the origin, where $\mathcal{A}_{r}=\mathcal{A} \backslash D(U, K)$ and $D(U, K)$ is the diagram of the pair $(U, K)$, i.e. the set of all $H \in \mathcal{A}$ such that $\alpha(H) \in \pi \mathbf{Z}$ for some restricted root $\alpha . D(U, K)$ is the union of finitely many families of equispaced hyperplanes. The set $S_{U / K}=\Psi(K / M \times D(U, K))$ is called the singular set in $U / K$. The regular set $(U / K)_{r}$ is the complement $U / K \backslash S_{U / K}$. See [9] p.271. 
it is easy to derive the following integral formula related to the polar decomposition of $U$ :

$$
\int_{U} f(u) d u=c \int_{K \times \mathcal{Q}_{0} \times K} f\left(k_{1}(\exp h) k_{2}\right) d k_{2} J^{2}(h) d h d k_{1},
$$

where $c^{-1}$ is given by (5.28) (see [9] Prop, 1.19 p.385). In particular for $f$ right- $K$ invariant (5.31) reduces to (5.26) (upon using (5.30) for the integral in $d k_{1}$ ).

In view of the polar decomposition of $U / K$ and remembering (5.22), it is clear that the heat kernel (5.19) is completely determined from its restriction to a (fixed) maximal torus $A x_{0}$.

Particularly important are then the $\tau$-spherical functions (5.10) and their representative matrices (5.11). These matrices satisfy

$$
\Phi_{\tau}^{\lambda}\left(k_{1} u k_{2}\right)^{a \xi}{ }_{b \xi^{\prime}}=\sum_{c, d} \tau\left(k_{1}\right)^{a}{ }_{c} \Phi_{\tau}^{\lambda}(u)^{c \xi}{ }_{d \xi^{\prime}} \tau\left(k_{2}\right)^{d}{ }_{b}, \quad u \in U, \quad k_{1}, k_{2} \in K,
$$

and are thus determined by their restriction to $A$.

A general procedure for calculating the $\tau$-spherical functions is the following. Consider the Casimir operator of $U, \Omega=\sum_{i} T_{i} T_{i}$, where $\left\{T_{i}\right\}$ is a given orthonormal basis of $\mathcal{U}$. $\Omega$ can be regarded as a second-order differential operator on $U$. The generalized radial part $\Omega_{r}$ of $\Omega$, acting on the restrictions $\left.\Phi_{\tau}^{\lambda}\right|_{A}$ is defined by

$$
\left.\left(\Omega \Phi_{\tau}^{\lambda}\right)\right|_{A}=\Omega_{r}\left(\left.\Phi_{\tau}^{\lambda}\right|_{A}\right)
$$

It can be shown that $\Omega_{r}$ is a well defined differential operator on $A$, whose coefficients are operator-valued functions on $A$ acting on $\Phi_{\tau}^{\lambda}(a)$ both from the left and from the right. The explicit form of $\Omega_{r}$ acting on arbitrary $\tau$-spherical functions was first calculated by Harish-Chandra. It is given e.g. in ref. [19] vol. II p. 277, where one needs to analytically continue from the noncompact symmetric space $G / K$ to the compact dual space $U / K$.

Now the Casimir operator acts as scalar multiplication on the operator-valued functions $u \rightarrow U^{\lambda}(u), \lambda \in \hat{U}$. Therefore the functions $\Phi_{\tau}^{\lambda}(a)$ are eigenfunctions of $\Omega_{r}$ and satisfy a certain differential equation on $A$. If $\operatorname{dim} A=1$ this differential equation reduces to an ordinary differential equation with operator coefficients, which, in principle, can be solved explicitly in terms of hypergeometric functions.

We shall now apply these considerations to Dirac spinors on $S^{N}$. 


\subsection{Spinors on $S^{N}$}

Let $U=\operatorname{Spin}(N+1), K=\operatorname{Spin}(N), U / K=S^{N}$.

Case 1. $N$ even. We apply the results obtained above to the fundamental spinor representations $\tau_{+}$and $\tau_{-}$of $\operatorname{Spin}(N)$ (cf. (2.15)). By using the well-known branching rule for $\operatorname{Spin}(N+1) \supset \operatorname{Spin}(N)$ (see e.g. [1]) we find

$$
\hat{U}\left(\tau_{+}\right)=\hat{U}\left(\tau_{-}\right)=\left\{\lambda_{n} \equiv\left(n+\frac{1}{2}, \frac{1}{2}, \ldots, \frac{1}{2}\right), \quad n=0,1, \ldots\right\} .
$$

The irreps $\lambda_{n}$ are called the spinor representations of $\operatorname{Spin}(N+1)$. As mentioned above, the multiplicity of $\tau_{ \pm}$in $\left.\lambda_{n}\right|_{K}$ is 1 for all $n$.

A Dirac spinor $\psi$ is a section of the (reducible) vector bundle $E^{\tau}=E^{\tau_{+}} \oplus E^{\tau_{-}}$defined by $\tau=\tau_{+} \oplus \tau_{-}$, i.e. $\psi \in \Gamma\left(E^{\tau}\right)$. We have the following relation between the iterated Dirac operator and the spinor Laplacian on a manifold $M$ :

$$
\nabla^{2}=\sum_{a=1}^{N} \nabla^{a} \nabla_{a}-R / 4
$$

where $R$ is curvature scalar of $M$. For $M=S^{N}$ we have $R=N(N-1)$. Thus the eigenvalues $\lambda_{n, N}^{2}$ of $-\nabla^{2}$ on $S^{N}$ are related to the eigenvalues $\omega_{n}$ of $-\sum_{a} \nabla^{a} \nabla_{a}$ by

$$
\lambda_{n, N}^{2}=\omega_{n}+N(N-1) / 4 .
$$

Now from eq. (5.6) we have for $E^{\tau_{+}}$

$$
\omega_{n}=\omega_{\lambda_{n}}=C_{2}\left(\lambda_{n}\right)-C_{2}\left(\tau_{+}\right) .
$$

For $E^{\tau_{-}}$we get the same eigenvalues since $C_{2}\left(\tau_{+}\right)=C_{2}\left(\tau_{-}\right)$(see below).

The heat kernel of $\nabla^{2}$ (with one point at the origin) is the direct sum $K=K^{+} \oplus K^{-}$, where the heat kernels $K^{ \pm}$for $E^{\tau_{ \pm}}$are given, in operator notation, by (cf. (5.20))

$$
\begin{aligned}
K^{+}(x, t) & \equiv K^{+}\left(x_{0}, x, t\right)=\frac{1}{\Omega_{N} d_{\tau_{+}}} \sum_{n=0}^{\infty} d_{\lambda_{n}} \Phi_{+}^{n}(\sigma(x)) U\left(x_{0}, x\right) e^{-t \lambda_{n, N}^{2}}, \\
K^{-}(x, t) & \equiv K^{-}\left(x_{0}, x, t\right)=\frac{1}{\Omega_{N} d_{\tau_{-}}} \sum_{n=0}^{\infty} d_{\lambda_{n}} \Phi_{-}^{n}(\sigma(x)) U\left(x_{0}, x\right) e^{-t \lambda_{n, N}^{2}} .
\end{aligned}
$$

The extra factor $1 / \Omega_{N}$ occurs here because we have chosen the normalization in which the total volume of $S^{N}$ is $\Omega_{N}$ (given by (3.42)) in order to compare our results with those of section 4. The $\Phi_{+}^{n}\left(\Phi_{-}^{n}\right)$ are the $\tau_{+}\left(\tau_{-}\right)$-spherical functions, i.e. the linear operators in $V_{\tau_{+}}\left(V_{\tau_{-}}\right)$defined by

$$
\Phi_{+}^{n}(u)=P_{\tau_{+}} U^{\lambda_{n}}(u) P_{\tau_{+}}, \quad u \in U
$$


and a similar relation for $\Phi_{-}^{n}$, where $P_{\tau_{ \pm}}$are the projectors of $V_{\lambda_{n}}$ onto the subspaces where $\left.\lambda_{n}\right|_{K}$ is equivalent to $\tau_{+}$and $\tau_{-}$, respectively. For $x, y$ nonconjugate points on $S^{N}$ $U(x, y)$ is the (vector-bundle) parallel transport operator from the fibre $E_{y}$ to the fibre $E_{x}$ along the shortest geodesic between $y$ and $x$. Therefore for each $x \in S^{N} \backslash\{$ south pole\}, the $\Phi_{ \pm}^{n}(\sigma(x)) U\left(x_{0}, x\right)$ in (5.38)-(5.39) are linear maps from $E_{x}$ to the fibre $E_{x_{0}} \simeq V_{\tau_{ \pm}}$at the origin (the north pole). This way the heat kernel $K(x, t) \equiv K\left(x_{0}, x, t\right)$ has the correct structure (recall that $K\left(x, x^{\prime}, t\right) \in \operatorname{Hom}\left(E_{x^{\prime}}, E_{x}\right)$ ). It is easy to see that the functions $x \rightarrow \Phi_{ \pm}^{n}(\sigma(x)) U\left(x_{0}, x\right)$ have a well defined limit as $x$ approaches the south pole, so that the heat kernel is regular everywhere (cf. (5.17), see also later).

We now need to compute $C_{2}\left(\lambda_{n}\right), C_{2}\left(\tau_{ \pm}\right), d_{\lambda_{n}}, d_{\tau_{ \pm}}$and $\Phi_{ \pm}^{n}$. The Casimir number of an irrep with highest weight $\lambda$ is given by Freudenthal's formula:

$$
C_{2}(\lambda)=(\lambda+\rho)^{2}-\rho^{2}=\lambda \cdot(\lambda+2 \rho)
$$

where $\rho$ is half the sum of the positive roots of the group. $\rho$ is also given by

$$
\rho=w_{1}+w_{2}+\ldots+w_{r}
$$

where $\left\{w_{j}\right\}_{j=1, \ldots, r}$ are the fundamental weights ( $r$ is the rank of the group). The fundamental weights of $U=\operatorname{Spin}(N+1)$ ( $N$ even) are given by (4) p.224 of [1]. Thus the element $\rho$ for $U$ is

$$
\rho=\left(\frac{N-1}{2}, \frac{N-3}{2}, \ldots, \frac{3}{2}, \frac{1}{2}\right) .
$$

Using this and (5.34) in (5.41) we find

$$
C_{2}\left(\lambda_{n}\right)=(n+N / 2)^{2}-N(N-1) / 8 .
$$

The calculation of $C_{2}\left(\tau_{ \pm}\right)$is similar. The fundamental weights of $K=\operatorname{Spin}(N)(N$ even) are given by (5) p.224 of [1]. Thus the $\rho$ element for $K$ is

$$
\rho=\left(\frac{N}{2}-1, \frac{N}{2}-2, \ldots, 1,0\right) .
$$

Using this and (2.15) in (5.41) gives

$$
C_{2}\left(\tau_{+}\right)=C_{2}\left(\tau_{-}\right)=N(N-1) / 8
$$

Using (5.44) and (5.46) in (5.37) and (5.36) we find

$$
\begin{aligned}
\omega_{n} & =(n+N / 2)^{2}-N(N-1) / 4, \\
\lambda_{n, N}^{2} & =(n+N / 2)^{2},
\end{aligned}
$$

in complete agreement with (3.26). 
Concerning the dimensions, these are given by the Weyl formula

$$
d_{\lambda}=\prod_{\alpha>0} \frac{\alpha \cdot(\lambda+\rho)}{\alpha \cdot \rho},
$$

where the product is over the positive roots $\alpha$ of the group. For $U=\operatorname{Spin}(N+1)(N$ even) the positive roots are given by

$$
\left\{\mathbf{e}_{i}\right\}_{i=1, \ldots, N / 2}, \quad\left\{\mathbf{e}_{i}+\mathbf{e}_{j}, \mathbf{e}_{i}-\mathbf{e}_{j}\right\}_{1 \leq i<j \leq N / 2}
$$

where $\left\{\mathbf{e}_{i}\right\}$ is the standard orthonormal basis of $\mathbf{R}^{N / 2}$. A simple calculation gives the dimensions of the spinor representations of $\operatorname{Spin}(N+1)$ as

$$
d_{\lambda_{n}}=\frac{2^{N / 2}(N+n-1) !}{n !(N-1) !}
$$

in agreement with (3.44). In a similar way we calculate

$$
d_{\tau_{+}}=d_{\tau_{-}}=2^{N / 2-1}
$$

as claimed in section 2. Finally let us determine $\Phi_{+}^{n}$.

As mentioned before, since

$$
\Phi_{+}^{n}\left(k_{1} u k_{2}\right)=\tau_{+}\left(k_{1}\right) \Phi_{+}^{n}(u) \tau_{+}\left(k_{2}\right), \quad u \in U, \quad k_{1}, k_{2} \in K
$$

it is enough to calculate the restrictions $\Phi_{+}^{n}(a), a \in A$. In our case $A$ is one-dimensional (thus $A \simeq S^{1}$ ) and it is well known that $M$, the centralizer of $A$ in $K$, may be identified with $\operatorname{Spin}(N-1)$. From the relation $a m=m a(a \in A, m \in M)$ and from (5.53) we have

$$
\Phi_{+}^{n}(a) \tau_{+}(m)=\Phi_{+}^{n}(a m)=\Phi_{+}^{n}(m a)=\tau_{+}(m) \Phi_{+}^{n}(a), \quad m \in M, a \in A,
$$

i.e., the operators $\Phi_{+}^{n}(a), a \in A$, commute with all the operators of the representation $\left.\tau_{+}\right|_{M}$. But from the branching rule for $\operatorname{Spin}(N) \supset \operatorname{Spin}(N-1)$ we immediately find that $\left.\tau_{+}\right|_{M}=\sigma$, the unique fundamental spinor representation of $\operatorname{Spin}(N-1)$. Since $\sigma$ is irreducible, it follows from Schur's lemma that $\Phi_{+}^{n}(a)$ must be proportional to the identity operator in $V_{\tau_{+}}$,

$$
\Phi_{+}^{n}(a)=f_{n}(a) \mathbf{1}, \quad a \in A
$$

where $f_{n}$ is a scalar function on $A$.

Let $H$ be the element of $\mathcal{A}$ satisfying $\alpha(H)=1$, where $\alpha$ is the unique positive restricted root of $U / K$. Let us normalize the scalar product $<,>$ on $\mathcal{A}$ (induced from that on $\mathcal{P}$ ) so that $\langle H, H\rangle=1$. For $a \in A$ we write $a=a_{\theta}=\exp (\theta H)$, where $\theta \in \mathbf{R}$. If $d\left(x, x^{\prime}\right)$ denotes the geodesic distance between $x, x^{\prime} \in S^{N}$, then

$$
d\left(a_{\theta} x_{0}, x_{0}\right)=d\left(k a_{\theta} x_{0}, x_{0}\right)=\theta, \quad k \in K,
$$


where $x_{0}$ is the origin (in our case the north pole). It is clear that $\theta$ can be taken as a coordinate on $A$ (or on $A x_{0}$ ), and we can regard $f_{n}\left(a_{\theta}\right)$ as a function of $\theta$. Let us denote this function by the same symbol, i.e. $f_{n}(\theta)$. We shall now prove that

$$
f_{n}(\theta)=\phi_{n}(\theta)
$$

where $\phi_{n}(\theta)$ is given by (4.13).

In order to do this, we use the explicit formula for the generalized radial part of the Casimir operator acting on the functions $\Phi_{+}^{n}(a)$. The general formula in [19] vol. II p.277 has been specialized to the rank-one case by Thieleker in [16 (see also [18 p.281). For $U / K=S^{N}$ the formula simplifies further as there is only one positive restricted root $\alpha$ (with multiplicity $m_{\alpha}=N-1$ ). Thus let $\Omega_{M}, \Omega_{K}$ and $\Omega_{U}$ denote the following elements of the universal enveloping algebra of $\mathcal{U}$ :

$$
\begin{aligned}
\Omega_{M} & =\sum_{i=1}^{\operatorname{dim} M} W_{i}^{2} \\
\Omega_{K} & =\Omega_{M}+\sum_{i=1}^{m_{\alpha}} X_{\alpha i}^{2} \\
\Omega_{U} & =H^{2}+\Omega_{K}+\sum_{i=1}^{m_{\alpha}} Y_{\alpha i}^{2} .
\end{aligned}
$$

Here $\left\{W_{i}\right\}$ is an orthonormal basis of $\mathcal{M}$ (the Lie algebra of $\left.M\right),\left\{X_{\alpha i}\right\},\left\{Y_{\alpha i}\right\}$ are orthonormal bases of $\mathcal{K}_{\alpha}$ and $\mathcal{P}_{\alpha}$ respectively, where

$$
\begin{gathered}
\mathcal{K}_{\alpha}=\left\{X \in \mathcal{K}:(\text { ad } h)^{2} X=-\alpha(h)^{2} X, \quad \forall h \in \mathcal{A}\right\} \\
\mathcal{P}_{\alpha}=\left\{Y \in \mathcal{P}:(\text { ad } h)^{2} Y=-\alpha(h)^{2} Y, \quad \forall h \in \mathcal{A}\right\} .
\end{gathered}
$$

Since $\mathcal{U}=\mathcal{K} \oplus \mathcal{P}$, and

$$
\mathcal{K}=\mathcal{K}_{\alpha} \oplus \mathcal{M}, \quad \mathcal{P}=\mathcal{P}_{\alpha} \oplus \mathcal{A},
$$

it is clear that $\Omega_{M}, \Omega_{K}$ and $\Omega_{U}$ are simply the Casimir elements of $M, K$, and $U$, respectively.

If $u \rightarrow \pi(u)$ is a differentiable representation of a compact Lie group $U$ on a vector space $V$, we denote by $d \pi$ the corresponding representation of the Lie algebra $\mathcal{U}$ on $V$. Thus

$$
\pi(\exp X)=\exp (d \pi(X)), \quad X \in \mathcal{U} .
$$

We use the same symbol $d \pi$ to denote the uniquely defined extension of this representation to the universal enveloping algebra of $\mathcal{U}$. In order to simplify the notation we shall however omit the symbol $d \pi$ in the following. For instance, if $T_{1}, T_{2}$ are in the universal enveloping algebra we shall write $T_{1} \pi(u) T_{2}$ in place of $d \pi\left(T_{1}\right) \pi(u) d \pi\left(T_{2}\right)$. Thus in (5.65) 
$\Omega_{M}, \Omega_{K}$ and $X_{\alpha i}$ denote $d U^{\lambda}\left(\Omega_{M}\right), d U^{\lambda}\left(\Omega_{K}\right)$ and $d U^{\lambda}\left(X_{\alpha i}\right)$ respectively, whereas in (5.67)-(5.69) they denote $d \tau_{+}\left(\Omega_{M}\right), d \tau_{+}\left(\Omega_{K}\right)$ and $d \tau_{+}\left(X_{\alpha i}\right)$.

Now let $U=\operatorname{Spin}(N+1)$. For $\lambda \in \hat{U}$ let $U^{\lambda}(u)(u \in U)$ denote the operators of the irrep $\lambda$ on the vector space $V_{\lambda}$. Consider the operator-valued function on $\mathbf{R}$ defined by $\theta \rightarrow h_{\lambda}(\theta)=U^{\lambda}\left(a_{\theta}\right)$. Using Lemma 2 of [16] (with a proper analytic continuation to the compact case, i.e. with $t \rightarrow i \theta$ ) we find that $h_{\lambda}(\theta)$ satisfies the following differential equation

$$
\begin{array}{r}
\left(\frac{d^{2}}{d \theta^{2}}+m_{\alpha} \cot \theta \frac{d}{d \theta}\right) h_{\lambda}(\theta)+h_{\lambda}(\theta) \Omega_{M} \\
-\frac{1}{\sin ^{2} \theta}\left[\left(\Omega_{M}-\Omega_{K}\right) h_{\lambda}(\theta)+h_{\lambda}(\theta)\left(\Omega_{M}-\Omega_{K}\right)\right. \\
\left.+2 \cos \theta \sum_{i=1}^{m_{\alpha}} X_{\alpha i} h_{\lambda}(\theta) X_{\alpha i}\right] \\
=\Omega_{U} h_{\lambda}(\theta)=-C_{2}(\lambda) h_{\lambda}(\theta) .
\end{array}
$$

Now let $\lambda \in \hat{U}\left(\tau_{+}\right)$, i.e. $\lambda=\lambda_{n}$, and let $\Phi_{+}^{n}\left(a_{\theta}\right)$ be the $\tau_{+}$-spherical function $P_{\tau_{+}} h_{\lambda_{n}}(\theta) P_{\tau_{+}}$. By acting with $P_{\tau_{+}}$both from the left and from the right in (5.65) we obtain an equation for $\Phi_{+}^{n}\left(a_{\theta}\right)$. Observe that for $T_{1}$ and $T_{2}$ in the universal enveloping algebra of $\mathcal{K}$ we have (see [16] lemma 7 )

$$
P_{\tau_{+}} T_{1} h_{\lambda_{n}}(\theta) T_{2} P_{\tau_{+}}=T_{1} \Phi_{+}^{n}\left(a_{\theta}\right) T_{2}
$$

(Here $T_{1}, T_{2}$ mean $d U^{\lambda_{n}}\left(T_{1}\right), d U^{\lambda_{n}}\left(T_{2}\right)$ in the left-hand side, and $d \tau_{+}\left(T_{1}\right), d \tau_{+}\left(T_{2}\right)$ in the right-hand side.)

It is easy to see that

$$
\begin{gathered}
\Omega_{K} \Phi_{+}^{n}\left(a_{\theta}\right)=\Phi_{+}^{n}\left(a_{\theta}\right) \Omega_{K}=-C_{2}\left(\tau_{+}\right) \Phi_{+}^{n}\left(a_{\theta}\right), \\
\Omega_{M} \Phi_{+}^{n}\left(a_{\theta}\right)=\Phi_{+}^{n}\left(a_{\theta}\right) \Omega_{M}=-C_{2}(\sigma) \Phi_{+}^{n}\left(a_{\theta}\right) .
\end{gathered}
$$

Furthermore since $\Phi_{+}^{n}\left(a_{\theta}\right)$ is just a scalar operator, we have

$$
\begin{array}{r}
\sum_{i=1}^{m_{\alpha}} X_{\alpha i} \Phi_{+}^{n}\left(a_{\theta}\right) X_{\alpha i}=\sum_{i=1}^{m_{\alpha}} X_{\alpha i} X_{\alpha i} \Phi_{+}^{n}\left(a_{\theta}\right) \\
=\left(\Omega_{K}-\Omega_{M}\right) \Phi_{+}^{n}\left(a_{\theta}\right)=\left(C_{2}(\sigma)-C_{2}\left(\tau_{+}\right)\right) \Phi_{+}^{n}\left(a_{\theta}\right) .
\end{array}
$$

Using these relations and (5.65) we find the following differential equation for the scalar function $f_{n}(\theta)$ :

$$
\begin{array}{r}
\left(\frac{d^{2}}{d \theta^{2}}+(N-1) \cot \theta \frac{d}{d \theta}\right) f_{n}(\theta)-C_{2}(\sigma) f_{n}(\theta) \\
-\frac{1}{\cos ^{2}(\theta / 2)}\left(C_{2}\left(\tau_{+}\right)-C_{2}(\sigma)\right) f_{n}(\theta) \\
=-C_{2}\left(\lambda_{n}\right) f_{n}(\theta)
\end{array}
$$


Now $C_{2}(\sigma)$ may be obtained from (5.46) by letting $N \rightarrow N-1$, i.e.

$$
C_{2}(\sigma)=(N-1)(N-2) / 8 .
$$

Using (5.44), (5.46) and (5.71) in (5.70) we obtain

$$
\begin{array}{r}
\left(\frac{d^{2}}{d \theta^{2}}+(N-1) \cot \theta \frac{d}{d \theta}\right) f_{n}(\theta)-\frac{N-1}{4 \cos ^{2}(\theta / 2)} f_{n}(\theta) \\
=\left(\frac{(N-1)^{2}}{4}-\left(n+\frac{N}{2}\right)^{2}\right) f_{n}(\theta) .
\end{array}
$$

This is precisely the differential equation satisfied by the functions $\phi_{n}(\theta)$ in (4.13). Obviously $\Phi_{+}^{n}(e)=1$, and $f_{n}$ must satisfy $f_{n}(0)=1$. Thus (5.57) is proved and $\Phi_{+}^{n}\left(a_{\theta}\right)=\phi_{n}(\theta)$ 1. Notice that the open set $\mathcal{Q}_{0}$ (cf. subsection 5.1) is $(0, \pi)$ in this case. Since $\Phi_{+}^{n}\left(a_{\pi}\right)=0$, the functions $x \rightarrow \Phi_{+}^{n}(\sigma(x)) U\left(x_{0}, x\right)$ (cf. (5.38)) are well-defined also at the south pole (where they vanish). By proceeding in a similar way with $\tau_{-}$we find the same expression for $\Phi_{-}^{n}\left(a_{\theta}\right)$, namely $\Phi_{-}^{n}\left(a_{\theta}\right)=\phi_{n}(\theta) \mathbf{1}$. Using these formulas in (5.38)-(5.39), with $x=a_{\theta} x_{0}, a_{\theta}=\exp (\theta H)=\sigma(x)$, we find

$$
K\left(a_{\theta} x_{0}, t\right)=U\left(x_{0}, a_{\theta} x_{0}\right) f_{N}(\theta, t),
$$

where $f_{N}(\theta, t)$ is given by (4.12). For a generic point $x=k a_{\theta} x_{0} \in S^{N}(k \in K)$ we have $K(x, t)=U\left(x_{0}, x\right) f_{N}(\theta, t)$. Using (5.73) and (5.22) we also find $K\left(k^{2} x_{0}, t\right)^{a}{ }_{b}=$ $K\left(a x_{0}, t\right)^{a}{ }_{b}(a \in A)$, i.e. the matrix representing $K(x, t) \equiv K\left(x_{0}, x, t\right)$ in the parallel transported bases $\left\{\boldsymbol{\theta}_{a}(x)\right\}$ and $\left\{\boldsymbol{\theta}_{a}\left(x_{0}\right)\right\}=\left\{\mathbf{v}_{a}\right\}$ (cf. subsection 5.1), and not just its trace, is a zonal function. The heat kernel $K(x, t)$ is well defined also at the "antipodal manifold" $x=a_{\pi} x_{0}$ (the south-pole), where it vanishes. Finally, the heat kernel $K\left(x, x^{\prime}, t\right)$ for arbitrary points $x, x^{\prime}$ is given by eq. (4.15).

Case 2. $N$ odd. We apply the results of subsection 5.1 to the fundamental spinor representation $\tau$ of $K=\operatorname{Spin}(N)$ (given by (2.22)). By using the branching rule for $\operatorname{Spin}(N+1) \supset \operatorname{Spin}(N)$ we find

$$
\hat{U}(\tau)=\left\{\lambda_{n}^{+}, n=0,1, \ldots\right\} \bigcup\left\{\lambda_{n}^{-}, n=0,1, \ldots\right\},
$$

where $\lambda_{n}^{+}$and $\lambda_{n}^{-}$are the spinor representations of $\operatorname{Spin}(N+1)$, with highest weights

$$
\lambda_{n}^{ \pm}=\left(n+\frac{1}{2}, \frac{1}{2}, \ldots, \frac{1}{2}, \pm \frac{1}{2}\right)
$$

By calculating the Casimir numbers and the dimensions of these irreps as before, we find that $C_{2}\left(\lambda_{n}^{+}\right)$is equal to $C_{2}\left(\lambda_{n}^{-}\right)$and is given by the right hand side of (5.44), and 
similarly $d_{\lambda_{n}^{+}}=d_{\lambda_{n}^{-}}$is given by the right hand side of (5.51) with $2^{N / 2} \rightarrow 2^{(N-1) / 2}$, in agreement with our results in section 3. Also $d_{\tau}=2^{(N-1) / 2}$ and $C_{2}(\tau)=N(N-1) / 8$. The eigenvalues $\lambda_{n, N}^{2}$ of $-\nabla^{2}$ are given by (5.36)-(5.37), and we obtain again (5.48).

Thus the heat kernel of $\nabla^{2}$ (with one point at the origin) takes the form (cf. (5.20))

$$
K(x, t) \equiv K\left(x_{0}, x, t\right)=\frac{1}{\Omega_{N} d_{\tau}} \sum_{n=0}^{\infty} d_{\lambda_{n}^{+}}\left(\Phi^{n+}(\sigma(x))+\Phi^{n-}(\sigma(x))\right) U\left(x_{0}, x\right) e^{-t \lambda_{n, N}^{2}},
$$

where the $\tau$-spherical functions $\Phi^{n+}$ and $\Phi^{n-}$ are defined by

$$
\Phi^{n+}(u)=P_{\tau} U^{\lambda_{n}^{+}}(u) P_{\tau}, \quad u \in U
$$

with an analogous relation for $\Phi^{n-}$. [As before $P_{\tau}$ projects each representation space onto the subspace of vectors which transform under $K$ according to $\tau$, and $U\left(x_{0}, x\right)$ is the parallel spinor propagator from $x$ to $x_{0}$.]

In order to calculate $\Phi^{n \pm}$ notice that the operators $\Phi^{n \pm}(a), a \in A$, commute with all the $\tau(m), m \in M \simeq \operatorname{Spin}(N-1)$ (the centralizer of $A$ in $K$ ). The branching rule for $\operatorname{Spin}(N) \supset \operatorname{Spin}(N-1)$ gives now $\left.\tau\right|_{M}=\sigma_{+} \oplus \sigma_{-}$, where $\sigma_{+}$and $\sigma_{-}$are the two fundamental spinor representations of $\operatorname{Spin}(N-1)(\mathrm{cf}$. (2.23)). Suppose we fix an orthonormal basis of $V_{\tau}$ adapted to the direct sum decomposition $V_{\tau}=V_{\sigma_{+}} \oplus V_{\sigma_{-}}$. By applying Schur lemma we have

$$
\begin{aligned}
& \Phi^{n+}(a)=f_{n}(a) \mathbf{1}_{+} \oplus \tilde{f}_{n}(a) \mathbf{1}_{-}, \quad a \in A, \\
& \Phi^{n-}(a)=g_{n}(a) \mathbf{1}_{+} \oplus \tilde{g}_{n}(a) \mathbf{1}_{-}, \quad a \in A,
\end{aligned}
$$

where $f_{n}, \tilde{f}_{n}, g_{n}$ and $\tilde{g}_{n}$ are scalar functions on $A$ and $\mathbf{1}_{ \pm}$denote the identity operators in $V_{\sigma_{ \pm}}$. We shall now determine these scalar functions by using the radial part of the Casimir operator together with the Schur orthogonality relations for the irreps of $U$.

As before, let $\alpha$ be the single positive restricted root of $S^{N}$, and write $a \in A$ as $a=a_{\theta}=\exp (\theta H), \theta \in \mathbf{R}$, where $H \in \mathcal{A}$ is chosen so that $<H, H>=\alpha(H)=1$. We consider $f_{n}$ etc. as functions of $\theta$ and denote them by the same symbols, i.e. $f_{n}(\theta)$ etc.

For $U=\operatorname{Spin}(N+1)$ and $\lambda \in \hat{U}$ let as before $h_{\lambda}(\theta)$ denote the operator valued function $\theta \rightarrow U^{\lambda}\left(a_{\theta}\right)$. Then $h_{\lambda}(\theta)$ satisfies again (5.65) (by Lemma 2 of [16]).

Now let $\lambda \in \hat{U}(\tau)$, i.e. $\lambda=\lambda_{n}^{+}$or $\lambda_{n}^{-}$. By acting with $P_{\tau}$ in (5.65) both from the left and from the right we obtain an equation for $\Phi^{n+}\left(a_{\theta}\right)$ and $\Phi^{n-}\left(a_{\theta}\right)$.

Since $C_{2}\left(\sigma_{+}\right)=C_{2}\left(\sigma_{-}\right)=(N-1)(N-2) / 8$, we have

$$
\left(\Omega_{M}-\Omega_{K}\right) \Phi^{n \pm}\left(a_{\theta}\right)=\Phi^{n \pm}\left(a_{\theta}\right)\left(\Omega_{M}-\Omega_{K}\right)=\left(C_{2}(\tau)-C_{2}\left(\sigma_{+}\right)\right) \Phi^{n \pm}\left(a_{\theta}\right) .
$$

However since $\Phi^{n \pm}\left(a_{\theta}\right)$ are not scalar operators, eq. (5.69) is no longer valid. For $p, q \in \mathbf{C}$ let $\operatorname{diag}(p, q)$ denote the operator $p \mathbf{1}_{+} \oplus q \mathbf{1}_{-}$in $V_{\tau}$. Then it is not difficult to 
show that

$$
\begin{aligned}
\sum_{i=1}^{N-1} X_{\alpha i} \operatorname{diag}(p, q) X_{\alpha i} & =-\left(C_{2}(\tau)-C_{2}\left(\sigma_{+}\right)\right) \operatorname{diag}(q, p) \\
& =-\frac{N-1}{4} \operatorname{diag}(q, p) .
\end{aligned}
$$

Take for example $N=3$. By writing

$$
S^{3} \simeq S U(2) \simeq S U(2) \times S U(2) / S U(2)_{\text {diag }}
$$

we have $U=S U(2) \times S U(2)$ and $K=S U(2)_{\text {diag }}$, the diagonal subgroup of $U$. Let $\mathcal{S}$ denote the Lie algebra of $S U(2) \simeq \operatorname{Spin}(3)$. Then $\mathcal{U}=\mathcal{S} \oplus \mathcal{S}$ and the subspaces $\mathcal{K}$ and $\mathcal{P}$ in the reductive decomposition $\mathcal{U}=\mathcal{K} \oplus \mathcal{P}$ are easily identified as

$$
\begin{gathered}
\mathcal{K}=\{(X, X), \quad X \in \mathcal{S}\}, \\
\mathcal{P}=\{(X,-X), \quad X \in \mathcal{S}\} .
\end{gathered}
$$

Take $\mathcal{A}=\mathbf{R}\left(T_{3},-T_{3}\right) \subset \mathcal{P}$, where $\left\{T_{1}, T_{2}, T_{3}\right\}$ is a basis of $\mathcal{S}$ satisfying the commutation relations

$$
\left[T_{1}, T_{2}\right]=T_{3}, \quad\left[T_{2}, T_{3}\right]=T_{1}, \quad\left[T_{3}, T_{1}\right]=T_{2} .
$$

Then $\left(a d T_{3}\right)^{2} T_{i}=-T_{i}(i=1,2)$ and comparing with (5.63) we see that the subspaces $\mathcal{K}_{\alpha}$ and $\mathcal{P}_{\alpha}$ are generated by $\left\{\left(T_{i}, T_{i}\right), i=1,2\right\}$ and $\left\{\left(T_{i},-T_{i}\right), \quad i=1,2\right\}$, respectively.

Now let $\tau$ be the $j=1 / 2$ fundamental spinor representation of $S U(2)_{\text {diag. }}$ Then

$$
d \tau\left(\left(T_{3}, T_{3}\right)\right)=-\frac{i}{2}\left(\begin{array}{cc}
1 & 0 \\
0 & -1
\end{array}\right)
$$

and

$$
\left.d \tau\left(X_{\alpha 1}\right)=d \tau\left(\left(T_{1}, T_{1}\right)\right)=-\frac{i}{2}\left(\begin{array}{ll}
0 & 1 \\
1 & 0
\end{array}\right), \quad d \tau\left(X_{\alpha 2}\right)=d \tau\left(\left(T_{2}, T_{2}\right)\right)\right)=\frac{1}{2}\left(\begin{array}{cc}
0 & -1 \\
1 & 0
\end{array}\right)
$$

where $i=\sqrt{-1}$. From this we find (omitting the symbol $d \tau$ for simplicity)

$$
\sum_{i=1}^{2} X_{\alpha i} \operatorname{diag}(p, q) X_{\alpha i}=-\frac{1}{2} \operatorname{diag}(q, p)
$$

which is eq. (5.81) for $N=3$. The proof for $N$ (odd) $>3$ is similar and will be omitted.

Let $\partial_{\theta} \equiv d / d \theta$. Using (5.80) and (5.81) in (5.65) we get the following set of coupled equations for the functions $f_{n}, \tilde{f}_{n}$ :

$$
\left\{\begin{array}{l}
{\left[\partial_{\theta}^{2}+(N-1) \cot \theta \partial_{\theta}-C_{2}\left(\sigma_{+}\right)\right] f_{n}-\frac{N-1}{2 \sin ^{2} \theta} f_{n}+\frac{(N-1) \cos \theta}{2 \sin ^{2} \theta} \tilde{f}_{n}=-C_{2}\left(\lambda_{n}^{+}\right) f_{n},} \\
{\left[\partial_{\theta}^{2}+(N-1) \cot \theta \partial_{\theta}-C_{2}\left(\sigma_{+}\right)\right] \tilde{f}_{n}-\frac{N-1}{2 \sin ^{2} \theta} \tilde{f}_{n}+\frac{(N-1) \cos \theta}{2 \sin ^{2} \theta} f_{n}=-C_{2}\left(\lambda_{n}^{+}\right) \tilde{f}_{n} .}
\end{array}\right.
$$


The same set of equations (with $f_{n} \rightarrow g_{n}$ and $\tilde{f}_{n} \rightarrow \tilde{g}_{n}$ ) is obtained for the irreps $\lambda=\lambda_{n}^{-}$.

By taking the sum and the difference of the two equations in (5.89), it is then easy to see that the function $f_{n}(\theta)+\tilde{f}_{n}(\theta)$ satisfies the same differential equation (5.72) as $\phi_{n}(\theta)$ (given by (4.13)), and the function $f_{n}(\theta)-\tilde{f}_{n}(\theta)$ satisfies the same equation as the function $\psi_{n}(\theta)$ given by

$$
\psi_{n}(\theta)=\frac{\psi_{n 0}(\theta)}{\phi_{n 0}(0)}=\frac{n ! \Gamma\left(\frac{N}{2}\right)}{\Gamma\left(n+\frac{N}{2}\right)} \sin \frac{\theta}{2} P_{n}^{\left(\frac{N}{2}, \frac{N}{2}-1\right)}(\cos \theta),
$$

in the notations of section 3. [The equation for $\psi_{n}$ is the same as (5.72) with the replacement $\cos ^{2} \theta / 2 \rightarrow \sin ^{2} \theta / 2$ in the third term of the left-hand side.]

Thus $f_{n}(\theta)+\tilde{f}_{n}(\theta) \propto \phi_{n}(\theta)$, and since $\phi_{n}(0)=f_{n}(0)=\tilde{f}_{n}(0)=1$ we have

$$
f_{n}(\theta)+\tilde{f}_{n}(\theta)=2 \phi_{n}(\theta)
$$

Similarly, we have

$$
f_{n}(\theta)-\tilde{f}_{n}(\theta)=2 c \psi_{n}(\theta)
$$

where $c$ is a (possibly complex) constant to be determined. Thus

$$
\begin{aligned}
& f_{n}(\theta)=\phi_{n}(\theta)+c \psi_{n}(\theta), \\
& \tilde{f}_{n}(\theta)=\phi_{n}(\theta)-c \psi_{n}(\theta) .
\end{aligned}
$$

For the functions $g_{n}$ and $\tilde{g}_{n}$ we obtain similarly

$$
\begin{gathered}
g_{n}(\theta)=\phi_{n}(\theta)+d \psi_{n}(\theta), \\
\tilde{g}_{n}(\theta)=\phi_{n}(\theta)-d \psi_{n}(\theta),
\end{gathered}
$$

where $d$ is another constant to be determined. Notice that necessarily $c \neq d$, otherwise the functions $\Phi^{n+}(u)$ and $\Phi^{n-}(u)$ would coincide for all $u$ in $U$, violating the Schur orthogonality relations for the matrix coefficients of $U^{\lambda_{n}^{ \pm}}$.

It is easy to see that $c$ and $d$ must be pure imaginary. Indeed the $\tau$-spherical functions $\Phi_{\tau}^{\lambda}(u)=P_{\tau} U^{\lambda}(u) P_{\tau}$ satisfy $\Phi_{\tau}^{\lambda}\left(u^{-1}\right)=\Phi_{\tau}^{\lambda}(u)^{\dagger}$ (since the operators $U^{\lambda}(u)$ are unitary). At $u=a_{\theta}=\exp (\theta H)$ this gives $f_{n}(-\theta)=\overline{f_{n}(\theta)}$ and a similar relation for $\tilde{f}_{n}$. Therefore if $f_{n}, \tilde{f}_{n}$ are real they must be even functions of $\theta$, if they are complex their real parts must be even and their imaginary parts must be odd. Then using (5.93)-(5.94) and noting that $\phi_{n}(-\theta)=\phi_{n}(\theta), \psi_{n}(-\theta)=-\psi_{n}(\theta)$, we see that $\bar{c}=-c$. In a similar way we find $\bar{d}=-d$.

In order to determine $|c|$ and $|d|$ we write down the Schur orthogonality relations for the matrix coefficients $U^{\lambda_{n}^{+}}(u)^{a \xi}{ }_{b \xi}$ and $U^{\lambda_{n}^{-}}(u)^{a \xi}{ }_{b \xi}$. Using the polar decomposition 
$u=k_{1} a k_{2}$ and the integral formula (5.31) we obtain the following three equations:

$$
\begin{array}{r}
\int_{0}^{\pi}\left[f_{n}(\theta) f_{n}(-\theta)+\tilde{f}_{n}(\theta) \tilde{f}_{n}(-\theta)\right](\sin \theta)^{N-1} d \theta=\frac{2 d_{\tau}}{d_{\lambda_{n}^{+}}} \frac{\Omega_{N}}{\Omega_{N-1}}, \\
\int_{0}^{\pi}\left[g_{n}(\theta) g_{n}(-\theta)+\tilde{g}_{n}(\theta) \tilde{g}_{n}(-\theta)\right](\sin \theta)^{N-1} d \theta=\frac{2 d_{\tau}}{d_{\lambda_{n}^{-}}} \frac{\Omega_{N}}{\Omega_{N-1}} \\
\int_{0}^{\pi}\left[f_{n}(\theta) g_{n}(-\theta)+\tilde{f}_{n}(\theta) \tilde{g}_{n}(-\theta)\right](\sin \theta)^{N-1} d \theta=0 .
\end{array}
$$

We have used here the fact that in the present normalization the constant $c^{-1}$ in $(5.28)$ is given by

$$
\int_{\mathcal{Q}_{0}} J^{2}(h) d h=\int_{0}^{\pi}(\sin \theta)^{N-1} d \theta=\Omega_{N} / \Omega_{N-1} .
$$

Now using the above equations to express $f_{n}$ etc. in terms of $\phi_{n}$ and $\psi_{n}$, and remembering that $\phi_{n}$ is even and $\psi_{n}$ is odd, we can rewrite (5.97)-(5.99) as

$$
\begin{array}{r}
\int_{0}^{\pi}\left(\phi_{n}^{2}-c^{2} \psi_{n}^{2}\right)(\sin \theta)^{N-1} d \theta=\frac{2 \Omega_{N}}{d_{n} \Omega_{N-1}}, \\
\int_{0}^{\pi}\left(\phi_{n}^{2}-d^{2} \psi_{n}^{2}\right)(\sin \theta)^{N-1} d \theta=\frac{2 \Omega_{N}}{d_{n} \Omega_{N-1}}, \\
\int_{0}^{\pi}\left(\phi_{n}^{2}-c d \psi_{n}^{2}\right)(\sin \theta)^{N-1} d \theta=0,
\end{array}
$$

where $d_{n}$ is given by (4.14). Now from (3.38) we have

$$
\int_{0}^{\pi} \phi_{n}^{2}(\theta)(\sin \theta)^{N-1} d \theta=\int_{0}^{\pi} \psi_{n}^{2}(\theta)(\sin \theta)^{N-1} d \theta=\frac{\Omega_{N}}{d_{n} \Omega_{N-1}} .
$$

Using this in (5.101)- 5.103 ) we see that $c^{2}=d^{2}=-1$ and $c d=1$, whence

$$
c=i=\sqrt{-1}, \quad d=-i
$$

We have obtained

$$
\begin{aligned}
& f_{n}=\phi_{n}+i \psi_{n}=\tilde{g}_{n} \\
& \tilde{f}_{n}=\phi_{n}-i \psi_{n}=g_{n}
\end{aligned}
$$

Notice that $\Phi^{n \pm}\left(a_{\pi}\right)= \pm i(-1)^{n} \operatorname{diag}(1,-1)$, i.e. the spherical functions do not vanish at $\theta=\pi$ in this case (but the heat kernel does).

Let us prove that the limit as $x$ approaches the south pole of the functions $\Phi^{n \pm}(\sigma(x)) U\left(x_{0}, x\right)$ (cf. (5.76) ) exists (i.e. it does not depend on the direction). Recall that the parallel transport operator $U\left(x_{0}, x\right)$ from $x=\operatorname{Exp} X\left(X \in \mathcal{P}_{0}\right)$ to the origin $x_{0}=N P$ (the north pole) along the geodesic $\exp (-t X) x(t \in[0,1])$ is the same as the linear map between the fibres $E_{x}$ and $E_{x_{0}}$ corresponding to the transvection 
$\exp (-X)=(\sigma(x))^{-1}$, see subsection 5.1. Thus $\Phi^{n \pm}(\sigma(x)) U\left(x_{0}, x\right)=\Phi^{n \pm}(\sigma(x))(\sigma(x))^{-1}$. Now notice that the functions on $U$ given by $u \rightarrow \Phi^{n \pm}(u) u^{-1} \in \operatorname{Hom}\left(E_{u x_{0}}, E_{x_{0}}\right)$ are invariant under $u \rightarrow u k(k \in K)$. Therefore the functions $\varphi^{n+}$ and $\varphi^{n-}$ on $U / K$ given by 1

$$
x=u x_{0} \rightarrow \varphi^{n \pm}(x) \equiv \Phi^{n \pm}(u) u^{-1} \in \operatorname{Hom}\left(E_{x}, E_{x_{0}}\right)
$$

are well defined for all $x \in U / K=S^{N}$, in particular for $x=a_{\pi} x_{0}=S P$ (the south pole). Since $\Phi^{n \pm}(\sigma(x))(\sigma(x))^{-1}=\varphi^{n \pm}(x)$ for $x \in S^{N} \backslash\{S P\}$, we have

$$
\lim _{x \rightarrow S P} \Phi^{n \pm}(\sigma(x)) U\left(x_{0}, x\right)=\varphi^{n \pm}(S P),
$$

and our claim is proved.

Now since $K$ fixes the south pole, we have a representation $k \rightarrow \tilde{\tau}(k)$ of $K$ on the fibre $E_{S P}$ at the south pole. If $u$ is any element of $U$ such that $u x_{0}=S P$, then $u^{-1} K u=K$ and

$$
\tilde{\tau}(k)=u \tau\left(u^{-1} k u\right) u^{-1}, \quad k \in K
$$

It is easy to see that the linear maps $\varphi^{n \pm}(S P) \in \operatorname{Hom}\left(E_{S P}, E_{N P}\right)$ intertwine $\tilde{\tau}$ and $\tau$, i.e.

$$
\varphi^{n \pm}(S P) \tilde{\tau}(k)=\tau(k) \varphi^{n \pm}(S P), \quad \forall k \in K .
$$

Indeed let $u$ be any element of $U$ such that $u x_{0}=S P$ and let $k \in K$. Then

$$
\begin{array}{r}
\varphi^{n \pm}(S P) \tilde{\tau}(k)=\Phi^{n \pm}(u) u^{-1} u \tau\left(u^{-1} k u\right) u^{-1} \\
=\Phi^{n \pm}(k u) u^{-1}=\tau(k) \Phi^{n \pm}(u) u^{-1} \\
=\tau(k) \varphi^{n \pm}(S P)
\end{array}
$$

as claimed.

Since $\Phi^{n \pm}\left(a_{\pi}\right)= \pm i(-1)^{n} \operatorname{diag}(1,-1)$, it is clear that the $\varphi^{n \pm}(S P)$ are nontrivial intertwining operators, so that $\tilde{\tau}$ is equivalent to $\tau$. This is always true in the odd dimensional case, namely one can show [using (5.109)] that for $N$ odd, given any $\tau \in \hat{K}$, the representation $\tilde{\tau}$ of $K$ on the fibre $E_{S P}$ of the homogeneous vector bundle $E^{\tau}$ over $S^{N}$ is always equivalent to $\tau$.

For $N$ even one can show that if $\tau$ is the irreducible representation of $K=\operatorname{Spin}(N)$ with highest weight $\left(p_{1}, p_{2}, \ldots, p_{N / 2}\right)$, then $\tilde{\tau}=\left(p_{1}, p_{2}, \ldots, p_{N / 2-1},-p_{N / 2}\right)$. For example in our spinor case we have $\tilde{\tau}_{+}=\tau_{-}$and $\tilde{\tau}_{-}=\tau_{+}(\operatorname{cf}$. (2.15) $)$. If $\Phi_{+}^{n}$ is the $\tau_{+}$-spherical function (5.40), the limit $\varphi_{+}^{n}(S P)=\lim _{x \rightarrow S P} \Phi_{+}^{n}(\sigma(x)) U\left(x_{0}, x\right)$ is again well defined and $\varphi_{+}^{n}(S P)$ intertwines $\tilde{\tau}_{+}$with $\tau_{+}$(same proof as above). However since $\tilde{\tau}_{+}=\tau_{-} \nsim \tau_{+}$, it follows from Schur lemma that $\varphi_{+}^{n}(S P)=0$. This explains our previous result that $\Phi_{+}^{n}\left(a_{\pi}\right)=0$ for $N$ even.

\footnotetext{
${ }^{7}$ In the notations of subsection $5.1, \varphi^{n \pm}(x)=\varphi_{\lambda_{n}^{ \pm}}\left(x_{0}, x\right)$, cf. eq. (5.14).
} 
Using (5.106)-(5.107) in (5.78)-(5.79) and then in (5.76) we find that the heat kernel $K\left(a_{\theta} x_{0}, t\right), a_{\theta}=\exp (\theta H)$, is given again by (5.73), and $K\left(x, x^{\prime}, t\right)$ is given by (4.15). Thus we find complete agreement with our results of section 4 . 


\subsection{Harmonic analysis on noncompact symmetric spaces}

Let $G$ be a noncompact semisimple Lie group with finite center, $K$ a maximal compact subgroup, and $G / K$ the associated Riemannian symmetric space of the noncompact type. The results obtained in subsection 5.1 can be generalized to homogeneous vector bundles $E^{\tau}$ over $G / K(\tau \in \hat{K})$. Harmonic analysis on $G / K$ is well understood in the case of scalars. For vector bundles $L^{2}$-harmonic analysis on $G / K$ may be reduced to $L^{2}$-harmonic analysis on the group $G$, by letting $L^{2}\left(G / K, E^{\tau}\right)$ sit in $L^{2}(G)$ in a natural way.

In the noncompact case $\hat{G}$ (the set of equivalence classes of irreducible unitary representations of $G$ ) is not completely known, in general. Fortunately, we do not need to know all of $\hat{G}$ in order to do harmonic analysis. Indeed a representation $\lambda$ in $\hat{G}$ may contain a given $\tau \in \hat{K}$ and still not appear in the direct integral decomposition (over $\hat{G}$ ) of the induced representation $\pi_{\tau}$ of $G$ on $L^{2}\left(G / K, E^{\tau}\right)$. In other words, the measure $d \mu(\lambda)$ in this decomposition (known as the Plancherel measure) has support in a proper subset of $\hat{G}$. The main problem is then the explicit determination of the Plancherel measure and of the tempered representations (i.e. those representations with non-zero Plancherel measure).

For semisimple Lie groups this problem has been solved completely by Harish-Chandra (see e.g. [12]). Thus, harmonic analysis for bundles on $G / K$ is, in principle, known. Notice that this is only true for $K$ compact, i.e. in the Riemannian case. For (pseudoRiemannian) symmetric spaces $G / H$, with $H$ noncompact, we may have a contribution to the analysis on $L^{2}(G / H)$ from representations of $G$ with zero Plancherel measure in $\hat{G}$ (the so-called "complementary series").

We shall not discuss these matters further here. The important point for us is that the heat kernel of the Laplacian acting on $L^{2}\left(G / K, E^{\tau}\right)$ may be expanded in terms of the $\tau$-spherical functions in a way which is similar to (5.20) with $\sum_{\lambda \in \hat{U}(\tau)} d_{\lambda} \rightarrow \int_{\hat{G}(\tau)} d \mu(\lambda)$, i.e.

$$
K(x, t) \equiv K\left(x_{0}, x, t\right)=\frac{1}{d_{\tau}} \int_{\hat{G}(\tau)} \varphi_{\tau}^{\lambda}(\sigma(x)) U\left(x_{0}, x\right) e^{-t \omega_{\lambda}} d \mu(\lambda) .
$$

The notations here are as follows. The $\tau$-spherical functions are the (operator-valued) functions $g \rightarrow \Phi_{\tau}^{\lambda}(g)$ on $G$ defined by

$$
\Phi_{\tau}^{\lambda}(g)=P_{\tau} U^{\lambda}(g) P_{\tau}, \quad g \in G,
$$

where $U^{\lambda}(g)$ denote the operators of the representation $\lambda \in \hat{G}(\tau)$ (this set has the same meaning as for the compact group $U$ ) in a Hilbert space $H_{\lambda}$, and $P_{\tau}$ is the projector of $H_{\lambda}$ onto $H_{\tau}=\oplus_{\xi=1}^{\xi_{\lambda}} V_{\xi} \simeq V_{\tau} \otimes \mathbf{C}^{\xi_{\lambda}}$, the subspace of vectors of $H_{\lambda}$ which transform under $K$ according to $\tau$. As before, $\xi_{\lambda}$ denotes the multiplicity of $\tau$ in $\left.\lambda\right|_{K}$, and $\varphi_{\tau}^{\lambda}(g)$ denotes the partial trace of $\Phi_{\tau}^{\lambda}(g)$ with respect to $\mathbf{C}^{\xi_{\lambda}}$ (cf. (5.12)). The operator $\Phi_{\tau}^{\lambda}(g)$ is regarded as an element of $\operatorname{Hom}\left(H_{\tau}, H_{\tau}\right)$ and $\varphi_{\tau}^{\lambda}(g)$ as an element of $\operatorname{Hom}\left(V_{\tau}, V_{\tau}\right)$. 
We write the reductive (Cartan) decomposition of the Lie algebra $\mathcal{G}$ of $G$ as $\mathcal{G}=\mathcal{K} \oplus \mathcal{P}$, where $\mathcal{K}$ is the Lie algebra of $K$ and $\mathcal{P}$ is a complementary $A d(K)$-invariant subspace which we choose as $\mathcal{K}^{\perp}$, the orthogonal complement of $\mathcal{K}$ in $\mathcal{G}$ with respect to the Killing form $B$ :

$$
B(X, Y)=\operatorname{Tr} a d(X) \operatorname{ad}(Y), \quad X, Y \in \mathcal{G} .
$$

We identify $\mathcal{P}$ with $T_{x_{0}}(G / K)$ and for $x=\operatorname{Exp} X \in G / K(X \in \mathcal{P})$ we write $\sigma(x)=$ $\exp (X)$, where as before $\operatorname{Exp}$ and $\exp$ denote the exponential mappings in $G / K$ and $G$, respectively.

As in the compact case $U\left(x_{0}, x\right)$ denotes the (vector-bundle) parallel transport operator from $x$ to $x_{0}$ along the geodesic between them. Since there are no points conjugate to $x_{0}, U\left(x_{0}, x\right)$ is well defined for any $x$ in $G / K$. If $x=\operatorname{Exp} X$ then $U\left(x_{0}, x\right)=\exp (-X)=$ $(\sigma(x))^{-1}$ as a linear map from the fibre $E_{x}$ to the fibre $E_{x_{0}} \simeq V_{\tau}$.

It is well known that the Killing form is positive definite on $\mathcal{P}$ and negative definite on $\mathcal{K}$. If $\left\{T_{i}\right\}$ is a basis of $\mathcal{G}$, let $g_{i j}=B\left(T_{i}, T_{j}\right)$, and let $g^{i j}$ denote the inverse of the matrix $g_{i j}$. The Casimir operator of $G$ is the following element of the universal enveloping algebra of $\mathcal{G}$ :

$$
\Omega_{G}=\sum_{i, j=1}^{\operatorname{dim} G} g^{i j} T_{i} T_{j} .
$$

Let $\left\{X_{i}\right\}$ be a basis of $\mathcal{K}$, orthonormal with respect to $-B$, and let $\left\{Y_{i}\right\}$ be a basis of $\mathcal{P}$, orthonormal with respect to $B$. Then

$$
\Omega_{G}=\sum_{i=1}^{\operatorname{dim} \mathcal{P}} Y_{i}^{2}-\sum_{i=1}^{\operatorname{dim} \mathcal{K}} X_{i}^{2} .
$$

Viewed as a differential operator on $\Gamma\left(E^{\tau}\right) \sum Y_{i}^{2}$ induces the vector-bundle Laplacian $L_{G / K}$, and as before $\sum X_{i}^{2}=\Omega_{K}$, the (negative definite) Casimir element of $K$. Thus

$$
L_{G / K}=\Omega_{G}+\Omega_{K} .
$$

The eigenvalues $-\omega_{\lambda}$ of $L_{G / K}$ acting on the vector-valued functions $g \rightarrow P_{\tau} U^{\lambda}\left(g^{-1}\right) v$ $\left(v \in H_{\lambda}, \lambda \in \hat{G}(\tau)\right)$ may then be related to the Casimir values of $\lambda$ and $\tau$. Since $\Omega_{G}$ is in the center of the universal enveloping algebra of $\mathcal{G}$, it acts as a scalar in $H_{\lambda}$. Denoting this scalar by $-C_{2}(\lambda)$, we have from (5.117)

$$
-\omega_{\lambda}=-C_{2}(\lambda)-C_{2}(\tau),
$$

to be compared to the compact relation (5.6). There is a well-known duality between the noncompact and the compact symmetric spaces. Consider the subspace $\mathcal{U}=\mathcal{K} \oplus i \mathcal{P}$ of the complexification $\mathcal{G}^{\mathbf{C}}$ of $\mathcal{G}$, where $i=\sqrt{-1}$. The Lie algebra $\mathcal{U}$ is called a compact form of $\mathcal{G}$. It is a compact Lie algebra since the Killing form $B$ is negative definite on $\mathcal{U}$. Let 
$U$ denote a simply connected compact Lie group with Lie algebra $\mathcal{U}$. Then $U / K$ is the compact symmetric space which is dual to $G / K$. It is clear that the radial parts of the Casimir operators on $G$ and $U$ (acting on $\tau$-spherical functions) will be related by analytic continuation through $h \rightarrow i h$, for $h \in \mathcal{A}$. This amounts to changing trigonometric with hyperbolic functions.

\subsection{Spinors on $H^{N}$}

Let $G=\operatorname{Spin}(N, 1), K=\operatorname{Spin}(N), G / K=H^{N}$.

Case 1. $N$ even. In this case the Plancherel measure in $\hat{G}$ has both a discrete and a continuous part. The tempered representations are the discrete series and the principal unitary series. Let $\tau=\tau_{+} \oplus \tau_{-}$(same notations as in subsection 5.2). From the branching rule for $\operatorname{Spin}(N, 1) \supset \operatorname{Spin}(N)$ we find that no discrete series contain $\tau$ (see [6]). Let $U_{(i \lambda, \sigma)}$ denote the unitary principal series representation labelled by $\lambda \in \mathbf{R}$ and $\sigma \in \hat{M}$, where $M=\operatorname{Spin}(N-1)$ is the centralizer of $A$ in $K$ and $G=K A N$ is an Iwasawa decomposition of $G$ [18, 19]. This is the representation of $G$ induced by the representation $D$ of a minimal parabolic subgroup $M A N$ given by

$$
D(\operatorname{man})=\sigma(m) e^{i \lambda(\log a)} .
$$

By Frobenius Reciprocity $\left.U_{(i \lambda, \sigma)}\right|_{K}$ contains $\tau$ if and only if $\left.\tau\right|_{M}$ contains $\sigma$. Thus we find that the unitary principal series containing both $\tau_{+}$and $\tau_{-}$are the $U_{(i \lambda, \sigma)}$ with $\sigma=\left(\frac{1}{2}, \ldots, \frac{1}{2}\right)$, the fundamental spinor representation of $M$. The Plancherel measure for this principal series is (see [6])

$$
\begin{gathered}
d \mu\left(U_{(i \lambda, \sigma)}\right)=\frac{2^{N-2}}{\pi \Omega_{N-1}} d_{\sigma}|C(\lambda)|^{-2} d \lambda, \\
C(\lambda)=\frac{2^{N-2} \Gamma(N / 2)}{\sqrt{\pi}} \frac{\Gamma\left(i \lambda+\frac{1}{2}\right)}{\Gamma\left(i \lambda+\frac{N}{2}\right)},
\end{gathered}
$$

where $d_{\sigma}=2^{N / 2-1}$ is the dimension of $\sigma$ and $\Omega_{N-1}$ is the volume of $S^{N-1}$ (cf. (3.42)). As in the scalar case, the spinor Harish-Chandra function $C(\lambda)$ is determined by the asymptotic form at infinity of the $\tau$-spherical functions $\Phi_{+}^{\lambda}$ and $\Phi_{-}^{\lambda}$, where $\Phi_{ \pm}^{\lambda}=$ $P_{\tau_{ \pm}} U_{(i \lambda, \sigma)} P_{\tau_{ \pm}}$. The radial part of the Casimir operator acting on the restrictions $\Phi_{ \pm}^{\lambda}\left(a_{y}\right)$, $a_{y}=\exp (y H) \in A$, may be obtained from (5.65) by letting $\theta \rightarrow i y$ and applying $P_{\tau_{+}}$ (or $P_{\tau_{-}}$) both from the left and from the right. Again $\Phi_{+}^{\lambda}\left(a_{y}\right)=\phi_{\lambda}(y) \mathbf{1}$, where $\phi_{\lambda}$ is a scalar function on $A$. The Casimir value $-C_{2}(\lambda)$ for $U_{(i \lambda, \sigma)}$ may be easily calculated and is given by

$$
-C_{2}(\lambda)=-\lambda^{2}-N(N-1) / 8
$$


It may be obtained from (5.44) by letting $n \rightarrow-i \lambda-N / 2$. Using (5.35), (5.46) and (5.118) we find that the eigenvalues of $\nabla^{2}$ are given by

$$
\nabla_{\lambda}^{2}=-\omega_{\lambda}-R / 4=-\lambda^{2}, \quad \lambda \in \mathbf{R}
$$

The differential equation satisfied by the scalar function $\phi_{\lambda}(y)$ is obtained from (5.72) by letting $\theta \rightarrow i y$ and $n \rightarrow-i \lambda-N / 2$. This may be put in hypergeometric form and we find

$$
\phi_{\lambda}(y)=\cosh \frac{y}{2} F\left(i \lambda+\frac{N}{2},-i \lambda+\frac{N}{2}, \frac{N}{2},-\sinh ^{2} \frac{y}{2}\right) .
$$

Similarly we find $\Phi_{-}^{\lambda}\left(a_{y}\right)=\phi_{\lambda}(y) \mathbf{1}$. Using the above formulas in (5.112) we find for the heat kernel $K=K^{+} \oplus K^{-}$,

$$
K^{+}(x, t)=K^{-}(x, t)=U\left(x_{0}, x\right) \frac{2^{N-3} \Gamma(N / 2)}{\pi^{N / 2+1}} \int_{0}^{+\infty} \phi_{\lambda}(y) e^{-t \lambda^{2}}|C(\lambda)|^{-2} d \lambda,
$$

where $y=d\left(x_{0}, x\right)$. This agrees with 4.25$)-(4.26)$.

Case 2. $N$ odd. In this case the group $G=\operatorname{Spin}(N, 1)$ has no discrete series and the Plancherel measure is purely continuous. Let $\tau$ be the irrep of $K$ given by (2.22). By Frobenius Reciprocity we find that the unitary principal series representations containing $\tau$ are $U_{\left(i \lambda, \sigma_{+}\right)}$and $U_{\left(i \lambda, \sigma_{-}\right)}$, where $\sigma_{ \pm}=\left(\frac{1}{2}, \ldots, \frac{1}{2}, \pm \frac{1}{2}\right)$ are the two fundamental spinor representations of $M=\operatorname{Spin}(N-1)$ (cf. (2.23)). The Plancherel measure $d \mu\left(U_{\left(i \lambda, \sigma_{+}\right)}\right)=$ $d \mu\left(U_{\left(i \lambda, \sigma_{-}\right)}\right)$is given again by (5.120) (with $d_{\sigma} \rightarrow d_{\sigma_{+}}=2^{(N-3) / 2}$ ) and (5.121).

Since $\left.\tau\right|_{M}=\sigma_{+} \oplus \sigma_{-}$, the $\tau$-spherical functions $\Phi^{\lambda \pm}(g) \equiv P_{\tau} U_{\left(i \lambda, \sigma_{ \pm}\right)}(g) P_{\tau}$ at $g=a_{y}=$ $\exp (y H) \in A$ are given by (cf. (5.78)-(5.79))

$$
\begin{aligned}
& \Phi^{\lambda+}\left(a_{y}\right)=\operatorname{diag}\left(f_{\lambda}(y), \tilde{f}_{\lambda}(y)\right), \\
& \Phi^{\lambda-}\left(a_{y}\right)=\operatorname{diag}\left(g_{\lambda}(y), \tilde{g}_{\lambda}(y)\right) .
\end{aligned}
$$

The scalar functions $f_{\lambda}, \tilde{f}_{\lambda}, g_{\lambda}$ and $\tilde{g}_{\lambda}$ may be obtained respectively from $f_{n}, \tilde{f}_{n}, g_{n}$ and $\tilde{g}_{n}$ in (5.106)-(5.107) by letting $n \rightarrow-i \lambda-N / 2$ and changing the argument $\theta \rightarrow i y$. Thus

$$
\begin{aligned}
& f_{\lambda}=\phi_{\lambda}+i \psi_{\lambda}=\tilde{g}_{\lambda}, \\
& \tilde{f}_{\lambda}=\phi_{\lambda}-i \psi_{\lambda}=g_{\lambda}
\end{aligned}
$$

where $\phi_{\lambda}(y)$ is given by (5.124) and

$$
\psi_{\lambda}(y)=\frac{2 \lambda}{N} \sinh \frac{y}{2} F\left(i \lambda+\frac{N}{2},-i \lambda+\frac{N}{2}, \frac{N}{2}+1,-\sinh ^{2} \frac{y}{2}\right) .
$$

Using the above equations in (5.112) we find that the heat kernel $K(x, t)$ is given by the right-hand side of (5.125), again in agreement with our results in section 4 . 


\section{Acknowledgments}

The authors would like to thank Prof. Andrzej Trautman for useful discussions and for pointing out Ref. [17]. The work of A.H. was supported in part by the Schweizerisher Nationalfonds. 


\section{References}

[1] A.O. Barut and R. Raczka, Theory of Group Representations and Applications. (World Scientific, Singapore, 1986)

[2] R. Camporesi, Harmonic analysis and propagators on homogeneous spaces, Phys. Rep. 196 (1990) 1.

[3] R. Camporesi, The spinor heat kernel in maximally symmetric spaces, Commun. Math. Phys. 148 (1992) 283-308

[4] R. Camporesi and A. Higuchi, Spectral functions and zeta functions in hyperbolic spaces, J. Math. Phys. 35 (1994) 4217-4246

[5] R. Camporesi and A. Higuchi, The Plancherel measure for p-forms in real hyperbolic spaces, J. Geom. Phys. 15 (1994) 57-94

[6] R. Camporesi, The spherical transform for homogeneous vector bundles over hyperbolic spaces, preprint 1993

[7] R. Godement, A theory of spherical functions, Trans. Amer. Math. Soc. 73 (1952) 496-556

[8] I.S. Gradshteyn and I.M. Ryzhik, Tables of integrals, series and products, (rev. ed. Academic Press, New York, 1980).

[9] S. Helgason, Differential Geometry and Symmetric Spaces. (Academic Press, New York, 1962)

[10] S. Helgason, Differential Geometry, Lie Groups and Symmetric Spaces. (Academic Press, New York, 1978)

[11] S. Helgason, Groups and Geometric Analysis. (Academic Press, New York, 1984)

[12] A. Knapp, Representation Theory of Semisimple Groups, an Overview based on Examples. (Princeton University Press, 1986)

[13] S. Kobayashi and K. Nomizu, Foundations of Differential Geometry, vol. I and II (Interscience, New York, 1969).

[14] T.H. Koornwinder, A note on the multiplicity free reduction of certain orthogonal and unitary groups, Indag. Math. 44 (1982) 215-218 
[15] K. Nomizu, Invariant affine connections on homogeneous spaces, Amer. J. Math. 76 (1954) 33-65

[16] E. Thieleker, On the integrable and square-integrable representations of Spin(1,2m), Trans. Amer. Math. Soc. 230 (1977) 1-40

[17] A. Trautman, Spin structures on hypersurfaces and the spectrum of the Dirac operator on spheres, in: Spinors, Twistors, Clifford Algebras and Quantum Deformations (Kluwer Academic Publishers, 1993)

[18] N.R. Wallach, Harmonic Analysis on Homogeneous Spaces. (Marcel Dekker, New York, 1973)

[19] G. Warner, Harmonic Analysis on Semi-Simple Lie Groups, vol. I, II, (Springer Verlag, New York 1972) 